\title{
Complementary Functional Organization of Neuronal Activity Patterns in the Perirhinal, Lateral Entorhinal, and Medial Entorhinal Cortices
}

\author{
[CChristopher S. Keene, ${ }^{1}$ John Bladon, ${ }^{1}$ Sam McKenzie, ${ }^{2}$ Cindy D. Liu, ${ }^{1}$ Joseph 0'Keefe, ${ }^{1}$ and Howard Eichenbaum ${ }^{1}$ \\ ${ }^{1}$ Center for Memory and Brain, Boston University, Boston, Massachusetts 02215, and ${ }^{2}$ The Neuroscience Institute, New York University Langone Medical \\ Center, New York, New York 10016
}

It is commonly conceived that the cortical areas of the hippocampal region are functionally divided into the perirhinal cortex (PRC) and the lateral entorhinal cortex (LEC), which selectively process object information; and the medial entorhinal cortex (MEC), which selectively processes spatial information. Contrary to this notion, in rats performing a task that demands both object and spatial information processing, single neurons in PRC, LEC, and MEC, including those in both superficial and deep cortical areas and in grid, border, and head direction cells of MEC, have a highly similar range of selectivity to object and spatial dimensions of the task. By contrast, representational similarity analysis of population activity reveals a key distinction in the organization of information in these areas, such that PRC and LEC populations prioritize object over location information, whereas MEC populations prioritize location over object information. These findings bring to the hippocampal system a growing emphasis on population analyses as a powerful tool for characterizing neural representations supporting cognition and memory.

Key words: hippocampus; lateral entorhinal cortex; medial entorhinal cortex; memory; perirhinal cortex

\section{Significance Statement}

Contrary to the common view that brain regions in the "what" and "where" streams distinctly process object and spatial cues, respectively, we found that both streams encode both object and spatial information but distinctly organize memories for objects and space. Specifically, perirhinal cortex and lateral entorhinal cortex represent objects and, within the object-specific representations, the locations where they occur. Conversely, medial entorhinal cortex represents relevant locations and, within those spatial representations, the objects that occupy them. Furthermore, these findings reach beyond simple notions of perirhinal cortex and lateral entorhinal cortex neurons as object detectors and MEC neurons as position detectors, and point to a more complex organization of memory representations within the medial temporal lobe system.

\section{Introduction}

A main theme in the functional anatomy of the medial temporal lobe (MTL) memory system has emphasized input pathways composed of extensions of the classic "what" and "where" streams that converge within the MTL (Davachi, 2006; Diana et al., 2007; Eichenbaum et al., 2007; Witter et al., 2014). One pathway involves inputs from sensory and association areas that enter

\footnotetext{
Received Dec. 6, 2015; revised Jan. 25, 2016; accepted Feb. 16, 2016.

Author contributions: C.S.K., J.B., and H.E. designed research; C.S.K., J.B., C.D.L., and J.0. performed research; C.S.K., J.B., and S.M. analyzed data; C.S.K. and H.E. wrote the paper.

This work was supported by National Institute of Mental Health Grants MH-94263, MH051570, and ONR MURI N00014-10-1-0936.

The authors declare no competing financial interests.

Correspondence should be addressed to Dr. Howard Eichenbaum, Center for Memory and Brain, Boston University, Boston, MA 02215. E-mail: hbe@bu.edu.

DOI:10.1523/JNEUROSCI.4368-15.2016

Copyright $\odot 2016$ the authors $\quad 0270-6474 / 16 / 363660-16 \$ 15.00 / 0$
}

the MTL via the perirhinal cortex (PRC) and then the lateral entorhinal cortex (LEC) where information about objects and events is represented (Witter et al., 1989; Suzuki and Amaral, 1994; Burwell et al., 1995; Burwell and Amaral, 1998; Kerr et al., 2007; van Strien et al., 2009). The other pathway involves inputs from spatial processing areas that enter the MTL via the parahippocampal (in primates; postrhinal in rodents) cortex (PHC) and then the medial entorhinal cortex (MEC) where information about spatial context is represented. While there are connections between the PRC-LEC and PHC-MEC areas, most emphasize that the two streams converge primarily within the hippocampus where objects and events are represented in spatial context. This simple model has recently been challenged by reconsiderations of the functional distinctions between LEC and MEC that suggest a mixture of object and spatial processing functions in these areas (Knierim et al., 2013; Morrissey and Takehara-Nishiuchi, 2014; Sasaki et al., 2015). 
Here we examine object versus spatial coding in PRC, LEC, and MEC, respectively, as embodied in this model. Studies on MEC have highlighted "grid cell" neurons that fire with spatial periodicity throughout an environmental context (Hafting et al., 2005), as well as neurons that encode head direction and spatial borders (Sargolini et al., 2006; Witter et al., 2014). Notably, the observation of prominent spatial coding is almost exclusively from studies of animals foraging in an open field in the absence of salient nonspatial stimuli or task demands. By contrast, LEC neurons exhibit little spatial specificity in this behavioral situation (Hargreaves et al., 2005) and PRC neurons exhibit none (Deshmukh et al., 2012). Conversely, in experiments where animals perform object recognition or discrimination tasks, PRC and LEC neurons fire during the presentation of specific odor (in rats) or visual (in monkeys) cues, during maintenance of nonspatial memories, or associated with behavioral responses, but firing patterns associated with the animals' location in space were not examined (Suzuki et al., 1997; Young et al., 1997; Yanike et al., 2009; Igarashi et al., 2014; Ahn and Lee, 2015; Brown and Banks, 2015). Some recent studies have identified activity in PRC and LEC neurons associated with objects or visual patterns in particular places as animals explore an open field (Deshmukh and Knierim, 2011; Deshmukh et al., 2012; Tsao et al., 2013) or traverse a circular path in a maze (Burke et al., 2012; Neunuebel et al., 2013). In these studies, the objects and visual cues were not associated with specific behaviors or rewards, and the only behavioral demand was movement through the environment in which these cues were positioned. So, it is unclear whether the objects and visual cues were encoded as specific nonspatial stimuli independent of their locations or as among the stimuli composing distinct spatial representations of a changing environment.

In the present study, we examined the activity patterns of PRC, LEC, and MEC neurons and neuronal populations in animals performing a task in which they were required to use the current spatial context to associate objects with reward or nonreward at multiple locations within each context. This task demanded the animals to attend to the environmental cues and to the objects independent of their location in an environment, as well as to use the spatial context to guide retrieval of objectreward associations.

\section{Materials and Methods}

Subjects. Subjects were 10 male Long-Evans rats (Charles River) weighing between 300 and $325 \mathrm{~g}$ at the start of the experiment. All animals were single housed and maintained on a $12 \mathrm{~h}$ light/dark cycle (lights on 8:00 A.M. to 8:00 P.M.). Behavioral training and testing were conducted exclusively during the light phase. Animals were maintained at a minimum $85 \%$ of their ad libitum feeding body weight and had ad libitum access to water in the home cage. Procedures were conducted according to the requirements set by the National Institutes of Health and Boston University Institutional Animal Care and Use Committee.

Materials and apparatus. The behavioral training environment was a custom-built apparatus $(1601 \times 60 \mathrm{w} \times 40 \mathrm{~h} \mathrm{~cm})$ consisting of two 40 $\mathrm{cm} \times 40 \mathrm{~cm}$ boxes connected by a central alley. The training apparatus was surrounded by black curtains, which limited the availability of distal cues. Each context was composed of unique visual and tactile cues. The objects consisted of identical terra cotta pots $(10 \mathrm{~cm}$ high with an internal diameter of $9 \mathrm{~cm}$ ), with unique digging media and odors (e.g., purple beads with grapefruit scent). To prevent the animal being guided by the smell of the Froot Loop (Kellogg's), the pots and digging media were sprinkled with crushed Froot Loops. An open field environment $(1 \mathrm{~m} \times$ $1 \mathrm{~m}$ ) was used to examine spatial firing patterns of cells following behavioral training.

Behavioral task. Rats were trained to perform the complete behavioral paradigm through successive stages. Initially, rats were trained to dig for a reward (one-quarter Froot Loop) buried in a pot filled with unscented sand. Once rats reliably retrieved buried rewards, they were trained on a simple odor discrimination task in their home cages. Two pots filled with sand, each scented with a distinct odor (aloe and cloves), were simultaneously presented to the rat in pseudorandomized left or right positions. The aloe-scented pot was always rewarded, whereas the cloves-scented pot was never rewarded. Simple odor discrimination continued until the rats reached a criterion of $80 \%$ correct across 20 consecutive trials. Upon reaching criterion, rats were habituated to the testing apparatus during a 30 min exploration period with Froot Loops scattered throughout the environment and all context dividers removed. Following habituation, behavioral training in the complete task ensued.

The behavioral training environment consisted of two chambers composed of unique visual and tactile cues on the floors and walls connected by a central alleyway that allowed rats to shuttle between them, with access restricted to one context per trial (see Fig. 1). The objects consisted of identical terra cotta pots with unique digging media and odors (e.g., purple beads with grapefruit scent), which could be presented in either of two pseudorandomized positions for a given trial where the rat was restricted to a single context. Rewards in the form of one-quarter Froot Loop were buried in the rewarded pot on each trial. After reaching behavioral criterion, 10 rats were implanted with microdrives targeting PRC, LEC, or MEC (five PRC, four LEC, and four MEC with 3 microdrives yielding both PRC and LEC data in the same rats). Following recovery, neuronal activity in perirhinal or entorhinal cortex was monitored in rats during the retrieval of memories where two distinct environmental contexts ( 1 and 2 ) predicted different reward expectations for behavioral responses to two distinct objects (A and B) presented in either of two positions within each context. It is important to note that context dictates the opposing object-reward associations, whereas position within a context is irrelevant to the task demands. When presented at either position in Context 1, choosing Object A was rewarded and choosing Object B was not rewarded, whereas in Context 2, choosing Object B was rewarded and not Object A (see Fig. 1). Positions within a context shared the same object-reward association, whereas comparisons across context reflected opposing object-reward associations. Each session consisted of two contexts with two paired odor/digging media combinations, but new sets of contexts and stimuli were introduced throughout training in the same order across all rats. After each behavioral training session, rats were allowed to explore an open field environment while foraging for Froot Loops, which was later used to examine spatial firing patterns of cells in MEC.

Each session consisted of 90 trials, 45 in each context. In a typical trial, the animal was allowed to enter and explore one of the contexts in the absence of objects for $10 \mathrm{~s}$. At the end of this context exploration period, a divider was inserted in the middle of the context restricting the rat to one half of the context while two pots (Objects A and B) with unique digging media and odors were placed in the corners behind the divider. The divider was then removed, allowing the rat to approach the pots where it could choose to dig in the pot or refrain from digging and sample the other pot. The beginning of object sampling was defined as the moment in time when the rat's nose crossed the threshold of the pot rim. Object position within each context was pseudo-randomized, and no object occurred in the same location for more than three consecutive trials. After most trials, the animal moved into the opposite context via the alleyway. However, on 9 trials for each session, the animal remained in the same context for an additional trial to prevent utilization of a strict alternation strategy. On these trials, the pots were removed, the rat remained in the same context, and the context exploration period began, after which the divider was inserted and testing resumed as previously described. Before drive implantation, rats were trained to criterion, which was defined as correct performance on $70 \%$ of trials in each context over a 20 trial block. Following implantation, rats were retrained to criterion and given at least two overtraining sessions before initiation of electrophysiological recordings. As recordings progressed, rats were trained on new object-context association problems that involved novel digging mediums and contextual cues. Following the initial learning session in which criterion performance was obtained on a new problem, 
animals were presented with the same problem in a number of additional overtraining sessions, which provided the data for this study.

Surgery. Anesthesia was induced by inhalation of $5 \%$ isoflurane (Webster Veterinary Supply) in oxygen and was maintained at $2 \%-3 \%$ throughout surgery. Before surgery, animals were injected with the analgesic Buprenex (buprenorphine hydrochloride, $0.03 \mathrm{mg} / \mathrm{kg}$ i.m.; Reckitt Benckiser Healthcare), an antiobiotic cefazolin $(330 \mathrm{mg} / \mathrm{ml} \mathrm{i.m.;} \mathrm{West-}$ Ward Pharmaceutical), and placed in a stereotaxic frame (Kopf), where an incision was made along the midline to expose the skull. Animals were implanted with microdrives that contained 18-24 independently drivable tetrodes aimed at the junction of perirhinal and lateral entorhinal cortex (centered at anteroposterior $=-6.92 \mathrm{~mm}$; mediolateral $=5.2$ $\mathrm{mm} ; 16^{\circ}-20^{\circ}$ lateral angle, varied to more directly target LEC at $16^{\circ}$ and PRC at $20^{\circ}$ ) or the dorsocaudal portion of medial entorhinal cortex (anteroposterior $=-8.0 \mathrm{~mm}$; mediolateral $=4.6 \mathrm{~mm} ; 25^{\circ}$ angle from anterior to posterior; all coordinates derived from bregma). Each tetrode was composed of four $12 \mu \mathrm{m}$ RO 800 wires (Sandvik Kanthal HP Reid Precision Fine Tetrode Wire; Sandvik) gold-plated to reduce impedance to between 180 and $220 \mathrm{k} \Omega$ at $1 \mathrm{kHz}$. At the end of surgery, all tetrodes were lowered $\sim 5 \mathrm{~mm}$ and $\sim 2 \mathrm{~mm}$ into tissue, in PRC-LEC and MEC, respectively. In addition, animals were injected with postoperative doses of Buprenex and cefazolin as described above. Animals were allowed to recover for 1 week before behavioral testing resumed.

Neural recordings. Electrophysiological recordings were collected using a 96 channel OmniPlex D Neural Data Acquisition System (Plexon). Each channel was amplified and bandpass-filtered for both single-unit activity ( $154 \mathrm{~Hz}$ to $8.8 \mathrm{kHz}$ ) and local field potentials $(1.5 \mathrm{~Hz}$ to $400 \mathrm{kHz})$. Spike channels were referenced to a local electrode in the same region to remove movement-related noise. Action potentials were detected by threshold crossing and digitzed at $40 \mathrm{kHz}$. Cineplex Studio (Plexon) was used for video recording of behavioral training sessions. Single units were isolated using Offline Sorter (Plexon), and behavioral events were timestamped using Cineplex Editor (Plexon). All data analysis was performed using custom scripts for MATLAB (The MathWorks). To reduce the likelihood of recording from the same neurons across multiple sessions, tetrodes were lowered before each testing session $(\sim \geq 0.18 \mathrm{~mm})$, and the amount a tetrode was lowered was based on a visual inspection of the identified units. To maximize unit quality, a score of $1-10$ was generated for each unit based on the separation of each cluster from neighboring clusters and the background noise. Only units with a score of $\geq 5$ were included in the analysis presented here.

Histology. Upon completion of behavioral testing, rats were anesthetized with $<5 \%$ isoflurane in oxygen, and tetrode placements were confirmed by creating a lesion at the tetrode tip by passing a $40 \mu \mathrm{A}$ current until the connection was severed on each wire. Animals subsequently received an overdose injection of Euthanol (Virbac AH) and were perfused intracardially with $0.9 \%$ saline followed by $10 \%$ formalin phosphate (VWR). Brains were removed and placed in a $20 \%$ sucrose solution until processed. Using a cryostat (CM 3050s; Leica Biosystems), brains were cut into $40 \mu \mathrm{m}$ sections (coronal for PRC-LEC, sagittal for MEC), mounted onto presubbed glass slides, and stained with cresyl violet to determine the location of tetrode tip lesions and tetrode tracks. The stereotaxic atlas of Paxinos and Watson (2007) was used to confirm the localization of tetrode tip lesions within LEC and MEC. This information was used in conjunction with driver turn counts to estimate the neuronal layer for each recording, with each unit categorized as either superficial (layers II and III) or deep (layers IV-VI) for PRC, LEC, and MEC, as well as to distinguish between Areas 35 and 36 in PRC.

\section{Analysis}

Single-neuron analysis. Rats could sample the two objects presented on each trial multiple times, and all object sampling events in which the rat dug in the rewarded pot and refrained from digging in the nonrewarded pot were considered in our analysis. To estimate the entorhinal representation of every object sampling event, for each cell, the number of spikes fired was counted for up to the first $1.5 \mathrm{~s}$ of object sampling, and this count was divided by the sampling duration to give the average firing rate for each cell on each object sampling event.
Selectivity index (SI) for individual neurons. Firing rates during object sampling were calculated as the number of spikes as a function of the time from the onset of object sampling when the animal's snout begins to cover the rim of the pot until the animal began to dig or turn its head away from the pot, using event markers in the video recordings. As previously described (Komorowski et al., 2009), object, position, and context selectivity for each neuron were measured using an SI calculated as follows:

$$
S I=\frac{\left(n-\sum_{i=1}^{n}\left(\lambda_{i} / \lambda_{\text {pref }}\right)\right)}{n-1}
$$

where $n$ is the number of conditions for the dimension under study (e.g., 2 in the case of objects, 4 in the case of positions), $\lambda_{\mathrm{i}}$ is the average firing rate of the neuron for the $i$ th possible event type, and $\lambda_{\text {pref }}$ is the average firing rate of the neuron in the condition associated with highest firing rate for the dimension under study. SI $=1$ if a cell fired for only one condition. Conversely, SI $=0$ if the cell fired equally under all conditions. To test whether the SI values for individual units were larger than that expected by chance, we compared each observed SI value against a distribution of 10,000 SI scores in which the object and/or position identities of all events were randomly shuffled for each session individually. Cells were defined as significantly selective for a dimension if $p<0.01$ for the observed SI value relative to the shuffling distribution. This analysis was performed for each of four dimensions (see Fig. $5 A, B$ ): (1) for context SI, firing rates were compared between the two context conditions; (2) for position SI, firing rates were compared between the four position conditions; (3) for object SI, firing rates were compared between the two object conditions; and (4) for object-position SI, firing rates were compared among the eight object-position combinations (two objects in each of two positions in two contexts).

To further evaluate the observed average SI values for each dimension for each region, we used a similar bootstrap shuffling procedure. In this case, we shuffled the object and/or position identities for each trial 1000 times per session. Thus, the observed firing patterns were maintained, albeit with shuffled object and/or position identities. The average values of this shuffling distribution (see Fig. $5 \mathrm{~A}$, dotted lines) reflect a stringent criterion against which we compared the observed average SI values for each dimension for each region to determine significant coding for task dimensions. All post hoc analyses for SI comparisons used a Wilcoxon rank-sum test with a Bonferroni correction for multiple comparisons (significance threshold of $p=0.05 / 4$ dimensions $=0.0125$ ) to determine significance.

Characterization of spatial firing patterns of individual neurons. We additionally examined the spatial firing properties of MEC neurons as animals foraged in an open field, particularly to identify grid cells, border cells, and head direction cells. After each testing session, we continued to collect data from the same neurons (see Fig. 4, waveforms) as the animals foraged for randomly sprinkled Froot Loop bits in an open field environment over a period of $20 \mathrm{~min}$. Spatial firing rate maps were estimated using the total number of spikes that occurred when the rat was at a given location $(3 \times 3 \mathrm{~cm}$ bins $)$ divided by the total time spent in that bin. Only bins visited at least twice for a total time of at least $200 \mathrm{~ms}$ were included. Spikes were included if the rat was moving at a velocity $>3 \mathrm{~cm} / \mathrm{s}$. Spatial firing maps were smoothed with a 2D Gaussian filter $(\sigma=1$ pixel).

Grid scores were calculated using previously established methods (Brandon et al., 2011). Briefly, the six surrounding peaks of the autocorelogram of the smoothed rate map for each cell were identified, and the rate map was corrected for the ellipse of the hexagon. Then, the rotational symmetry of a circular donut of pixels encompassing the six identified peaks was calculated at $30,60,90,120$, and 180 degrees. The minimum difference in symmetry of 60 and 120 degrees to that at 30, 90, 120, and 180 degrees was then calculated as the raw grid score. A bootstrap confidence measure of the grid score was adopted (Bonnevie et al., 2013). We calculated the grid score as above for each cell 10,000 times after the spike timestamps were shuffled while preserving the temporal structure of the spike train. A unit was classified as a grid cell if the observed gridness 
score surpassed the critical threshold of $p=0.01$ compared with the shuffled distribution.

Similarly, border scores were calculated using methods described previously (Solstad et al., 2008). For each cell, its place fields were defined as contiguous groups of pixels in the smoothed rate map that had a firing rate of at least $30 \%$ of the maximum firing rate pixel. Then, the ratio of the place field's coverage of each wall to that place field's average distance from that wall was calculated to represent the border score. Border cells were designated as those with border scores $>0.4$ for any of the four walls.

To determine the animal's head direction, tracking coordinates for the LED were first smoothed to estimate the animal's location for any missing tracking frames. Then, the angle of displacement was calculated by taking the arc tangent of the difference in coordinates recorded at $40 \mathrm{~Hz}$, providing a heading estimate. As with grid cells, for each cell, the spike timestamps were shuffled 10,000 times while preserving the temporal structure of the spike train. The number of spikes at each head direction (in 5 degree bins) was then summed and divided by the total occupancy of that head direction. The length of the resultant vector was then compared with the shuffled distribution. Units with an observed vector length below the critical threshold of $p=0.01$ were considered head direction cells.

Representational similarity analysis (RSA) of neural ensemble activity patterns. Neural ensemble firing patterns were explored using an RSA to determine the extent to which multiple task dimensions were encoded by PRC, LEC, and MEC (Kriegeskorte et al., 2008; McKenzie et al., 2014). To measure the similarity of ensemble representations of different object sampling events, the average $z$-normalized firing rate for each neuron was calculated for all object sampling. A population vector was then composed for every sampling event based on these normalized rates, and the population vector of each event was then correlated to that for all other events, and then the correlation coefficients of similar comparisons (e.g., all comparisons between events involving different objects of the same value in the same position) were averaged, yielding a pattern of correlation coefficients that reflects the degree of similarity or separation of population representations between events that differ in multiple task dimensions, and these patterns were similar in each subject (see Fig. 6).

Using the pooled correlations from all sessions for all rats within a brain region (PRC, LEC, or MEC), we calculated the strength of the coding dimension (e.g., object in position) using the ensemble correlations. We first compared the average correlation for events within a given condition (e.g., all sampling events in the same position with Object A) for a task dimension versus events that were between conditions for that dimension (e.g., events in the same position with Object A vs Object B). In this manner, a single $\mathrm{d}^{\prime}$ was calculated for each dimension as follows:

$$
\mathrm{d}^{\prime}=\frac{\mu_{w}-\mu_{b}}{\sqrt{\frac{1}{2}\left(\sigma_{w}^{2}+\sigma_{b}^{2}\right)}}
$$

Where $\mu_{w}$ is the mean correlation coefficient for within-condition events for that dimension with variance, $\sigma_{w}^{2}$, and $\mu_{\mathrm{b}}$ is the mean correlation coefficient for between-condition events with variance, $\sigma_{b}^{2}$. The observed $\mathrm{d}^{\prime}$ was compared with bootstrapped data in which event identities were randomly shuffled 10,000 times, and then the correlation analysis and d' metric for each bootstrap sample were recomputed. When the observed $\mathrm{d}^{\prime}$ was $>95 \%$ of the 10,000 shuffled $\mathrm{d}^{\prime}$ metrics, the dimension captured by the $\mathrm{d}^{\prime}$ was considered to have been significantly coded by the perirhinal or entorhinal cortices.

We used the $\mathrm{d}^{\prime}$ metric to measure the separation of the distributions of the correlation coefficients for specific task features from 0 or against the distribution of coefficients from an appropriately opposing condition to characterize the population signal of that feature. Conjunctive objectposition coding was defined as the $\mathrm{d}^{\prime}$ distance between correlation coefficients for events that involved that same object in the same position versus events that involved different objects at the same position (see Fig. $\left.5 D, \mathrm{Obj}^{\star} \mathrm{Pos}\right)$. Object coding was defined as the $\mathrm{d}^{\prime}$ distance between correlation coefficients for events that involved the same object at different positions versus events that involved different objects at different positions within the same context (Fig. 5D, Object). Position coding was defined as the $\mathrm{d}^{\prime}$ distance between correlation coefficients for events at the same position versus events at different positions within the same context (Fig. 5D, Position). Context coding was defined as the $\mathrm{d}^{\prime}$ distance between correlation coefficients for comparisons among events in the same context at different positions versus that for all events in the opposing context (Fig. 5D, Context). All post hoc d' comparisons used a Bonferroni correction for multiple comparisons (significance threshold of $p=0.05 / 4$ dimensions $=0.0125$ ) to determine significance.

Cosine vector analysis. To ensure the robustness of the findings in the $\mathrm{d}^{\prime}$ distance analysis, ensemble representations were also analyzed using the cosine between population vectors to determine the similarity of representations. First, firing rates for each neuron were normalized to the maximum firing rate among all object-sampling events to create a population vector of normalized rates for each event. Then, a cosine score was calculated as 1 , the cosine of the included angle between points made by the pairs of vectors for the comparison of each event with all others of the same type. Strength of a coding dimension was calculated by comparing the mean cosine score for events within versus between sample conditions for that dimension. As with the RSA analysis, $\mathrm{d}^{\prime}$ distances were calculated for the cosine scores for the comparisons described in the previous paragraph.

Bayesian classifier. As a separate test of event similarity that had a different set of assumptions, a naive Bayesian classifier was used (MATLAB R2014b function with type set to determine the probability that a pattern of neural activity was recorded for each object and place combination, two objects in four positions; as in McKenzie et al., 2014). Because of to uneven sampling, rats often preferred a particular object and position combination and would sample those more often. Therefore, we only considered the last 5 sampling events for each object and place combination. When there were $<5$ events, that category of object and position trial was eliminated. Next, the $z$-scored population vector was calculated for each event, as described above. The dimensionality of the ensemble representation for each event was reduced via principal component analysis and only the first four components were used to categorize object/position combinations. Then, the mean, variance, and covariance of each object/position four-dimensional ensemble representation were estimated with one event missing from each object and position combination. Next, a multidimensional normal distribution with the estimated means, variance, and covariance matrices was fit to each cluster of object/position ensemble representations (maximum 8). Finally, the probability of the missing events being any of the possible object/position combinations was calculated based on the probability of that object/position combination given the ensemble representation as estimated by the normal distributions above.

The degree of dimensional coding was calculated in a similar fashion to that for the correlation coefficients and cosine analysis, although the $\mathrm{d}^{\prime}$ was calculated based on the probability of classifying within each dimension (e.g., events occurring at the correct position) or across that dimension (e.g., events occurring at a different position). To test significance, we ran a bootstrap analysis in which event identities were shuffled 10,000 times; and if the observed d' for the difference in probabilities was $>95 \%$ of the shuffled d' metrics, we concluded that PRC, LEC, or MEC encoded that dimension.

Temporal dynamics of population firing patterns. To assess when coding for different task dimensions emerged during object sampling, the $\mathrm{d}^{\prime}$ metric was calculated exactly as with the correlation RSA, except that $\mathrm{d}^{\prime}$ strength was calculated for $200 \mathrm{~ms}$ time bins centered \pm 3 s around object sampling (see Fig. 8). To tightly control for behavior during the approach to objects, we considered only the first object-sampling event of each trial. Significance testing of the observed $\mathrm{d}^{\prime}$ values was performed using the $\mathrm{d}^{\prime}$ bootstrap analysis at each time point with a significance threshold of $p=0.05$.

Dendrogram analysis. To explore the organization of ensemble representations for the 8 types of object sampling events (i.e., conjunctions of 2 objects in 2 positions within 2 contexts), we generated composite ensemble representations for each event type using the firing rates of all principal neurons recorded in PRC, LEC, and MEC. The composite population vector for each type of event was calculated as follows: for each neuron, the number of spikes observed was divided by the sampling duration on each event; then these firing rates were standardized into $z$-scores using the mean and SD of 


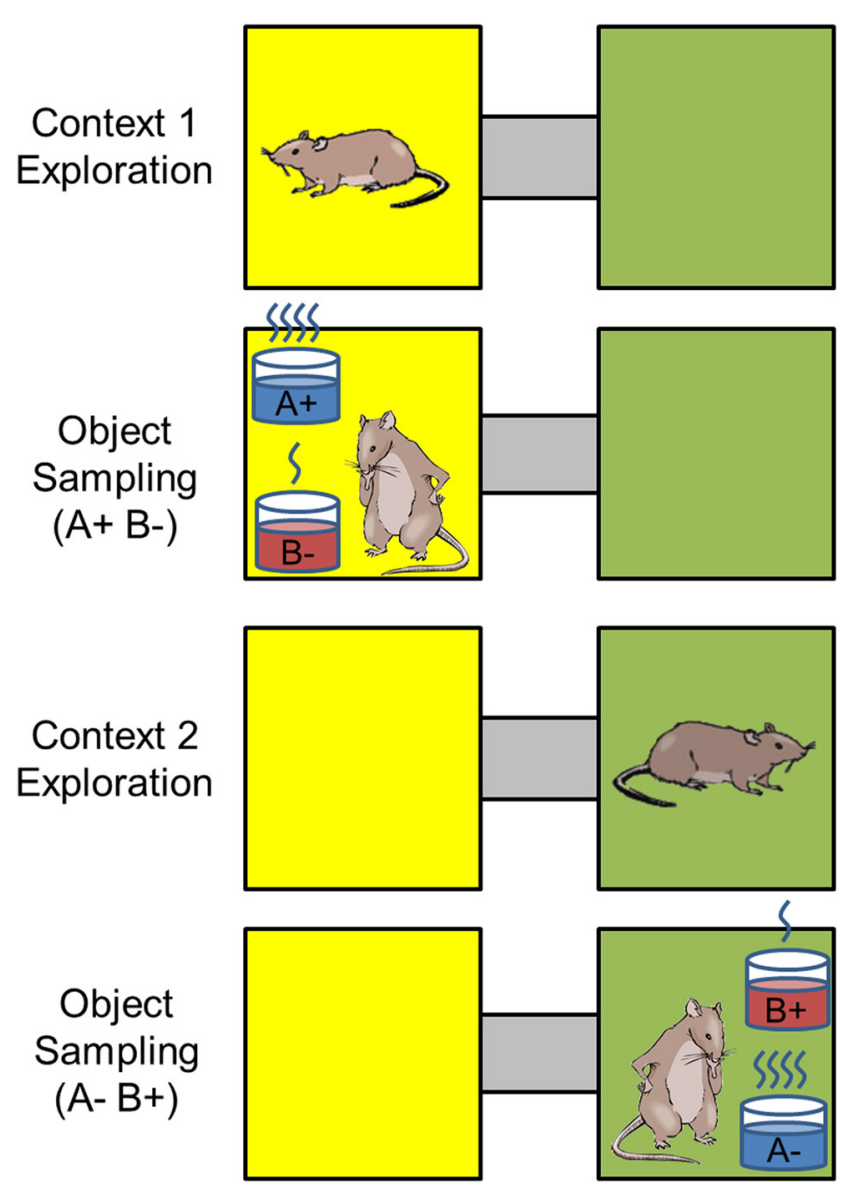

Figure 1. Task design. Rats were trained to perform a context-guided object association task. Each context consisted of unique tactile and visual stimuli. On a typical trial, rats were allowed to explore the context for $10 \mathrm{~s}$ (context exploration period). Then, two objects, consisting of terra cotta pots with unique digging media and odors, were presented in either of two positions (object sampling period). Object $A$, but not 0 bject $B$, was always reinforced in either position within Context 1 , whereas 0 bject $B$, but not 0 bject $A$, was always reinforced in Context 2.

firing rates across events; then the mean $z$-score firing rates across events were calculated. The relationships between the composite population vectors for the 8 types of events were then assessed using an agglomerative hierarchical clustering algorithm (MATLAB R2014b function 'linkage'). The agglomerative hierarchical clustering algorithm takes the unweighted average distance between pairs of the 8 vectors, where the Pearson's correlation coefficient was used as the distance metric. Of the 8 vectors, the two that were nearest were combined; then calculations of distances between the revised set of vectors were repeated and the nearest two vectors were combined. This process was repeated iteratively until only two combinations remained. The height of each line in the dendrogram represents the similarity (mean $r$ value) between the event types being connected.

\section{Results}

\section{Behavioral performance}

We recorded activity from neurons in PRC, LEC, and MEC while rats performed a context-guided object-reward association task that required subjects to select one of two objects presented simultaneously within each of two distinctive spatial contexts differing in multiple features (Fig. 1). When presented in Context 1, Object A was rewarded in either of two positions, and Object B was not. When presented in Context 2, Object B was rewarded in either position, and Object A was not. Context, but not position within context, was predictive of object-reward association in this task. Thus, subjects were required to use the current context to guide learning and retrieval of distinct associations for the same objects. Several different problems consisting of different contexts and objects were successively used (but will all be referred to here as Context 1 and 2 and Objects A and B). In the initial learning session on each problem, rats reached the performance criterion of $70 \%$ correct in each context within a 20 trial block on average by Trial 38 (range, 30-44 trials) for PRCimplanted rats $(n=5)$, by Trial 34 (range, $11-45$ trials) for LECimplanted rats $(n=4)$, and by Trial 38 (range, $17-70$ trials) for MEC-implanted rats $(n=4)$, respectively. A one-way ANOVA on the average trials to criterion revealed no differences in the rate at which rats learned new problems $\left(F_{(2,11)}=0.08, p=0.91\right)$. Subsequently, animals performed in overtraining sessions on each problem where performance was $96.4 \pm 1.0 \%$ for PRCimplanted rats, $97.0 \pm 0.8 \%$ for LEC-implanted rats, and $94.2 \pm$ $1.3 \%$ for MEC-implanted rats. A one-way ANOVA on performance in overtraining sessions revealed no differences $\left(F_{(2,58)}=\right.$ $1.49, p=0.23)$. In this study, data analysis focused on these overtraining sessions (14 for PRC, 17 for LEC, 30 for MEC).

\section{Single-neuron isolation}

In five PRC-implanted rats, a total of 204 units were isolated. In four LEC-implanted rats, a total of 188 units were isolated. In four MEC-implanted rats, a total of 323 units were isolated. A 10 $\mathrm{Hz}$ firing rate cutoff across the whole session was used to exclude interneurons, yielding 164 PRC neurons, 164 LEC neurons, and 236 MEC neurons for analysis (summarized in Table 1). The average scores for cluster quality were highly similar across the three regions $(\mathrm{PRC}=6.78 \pm 0.11 ; \mathrm{LEC}=6.93 \pm 0.11 ; \mathrm{MEC}=$ $6.76 \pm 0.09)$. Neurons were subdivided by whether they were recorded in superficial or deep laminae based on a combination of histological examination, driver turn counts, and maps of tetrode locations within the microdrives. The anatomical distribution of neurons recorded from PRC, LEC, and MEC is presented in Figure 2. Based on these criteria, a total of 92 deep units and 72 superficial units were identified in PRC, a total of 57 deep units and 107 superficial units were identified in LEC, and a total of 133 deep units and 103 superficial units were identified in MEC. In addition, a total of 95 units were identified in Area 35 and a total of 69 units were identified in Area 36 within PRC. Finally, open field exploration sessions following behavioral testing were used to characterize spatial firing properties of MEC units to further categorize and analyze spatially heterogeneous populations of grid cells, border cells, and head direction cells. A total of 23 grid cells, 27 border cells, and 125 head direction cells were isolated and analyzed separately from the other MEC units.

\section{PRC, LEC, and MEC neurons encode multiple dimensions of} both object and spatial information

During the object sampling period, neurons in PRC, LEC, and MEC exhibited remarkable mixed selectivity associated with multiple task dimensions both across the population of cells in each region and within single neurons. Several examples of this pattern are provided in Figure 3 where the firing pattern of each example is illustrated for each of the eight object-position combinations, four in each context. The beginning of object sampling was defined as the moment when the rat's nose reached the rim of the pot (the 0 time point for the rasters and histograms in Figs. 3, 4) and object sampling ended when the rat either began to dig or turned away, up to a maximum of $1.5 \mathrm{~s}$ after onset. Mixed selectivity in each brain region was readily apparent in neurons recorded in both superficial and deep layers, as well as within all the spatially heterogeneous subpopulations within MEC (summa- 
Table 1. Number and percentage of units having significant coding for specific task dimensions based on SI (interneurons excluded) and average SI \pm SEM ${ }^{a}$

\begin{tabular}{|c|c|c|c|c|c|c|c|c|}
\hline \multirow[b]{2}{*}{ Brain region } & \multicolumn{2}{|l|}{ Context } & \multicolumn{2}{|l|}{ Position } & \multicolumn{2}{|l|}{ Object } & \multicolumn{2}{|c|}{ 0bject $\times$ position } \\
\hline & No. significant & $\%$ & No. significant & $\%$ & No. signi & $\%$ & No. significant & $\%$ \\
\hline \multicolumn{9}{|l|}{ PRC } \\
\hline \multirow[t]{2}{*}{ Overall } & $49 / 164$ & $29.9 \%$ & $39 / 164$ & $23.8 \%$ & 27/164 & $16.5 \%$ & $46 / 164$ & $28.0 \%$ \\
\hline & \multicolumn{2}{|c|}{$0.33 \pm 0.02$} & \multicolumn{2}{|c|}{$0.43 \pm 0.02$} & \multicolumn{2}{|c|}{$0.31 \pm 0.02$} & \multicolumn{2}{|c|}{$0.55 \pm 0.02$} \\
\hline \multirow[t]{2}{*}{ Superficial } & $28 / 72$ & $38.9 \%$ & $24 / 72$ & $33.3 \%$ & $16 / 72$ & $22.2 \%$ & $26 / 72$ & $36.1 \%$ \\
\hline & \multicolumn{2}{|c|}{$0.36 \pm 0.03$} & \multicolumn{2}{|c|}{$0.45 \pm 0.03$} & \multicolumn{2}{|c|}{$0.33 \pm 0.03$} & \multicolumn{2}{|c|}{$0.58 \pm 0.02$} \\
\hline \multirow[t]{2}{*}{ Deep } & $21 / 92$ & $22.8 \%$ & $15 / 92$ & $16.3 \%$ & $11 / 92$ & $12.0 \%$ & $20 / 92$ & $21.7 \%$ \\
\hline & \multicolumn{2}{|c|}{$0.30 \pm 0.03$} & \multicolumn{2}{|c|}{$0.41 \pm 0.03$} & \multicolumn{2}{|c|}{$0.31 \pm 0.03$} & \multicolumn{2}{|c|}{$0.54 \pm 0.02$} \\
\hline \multirow[t]{2}{*}{ Area 35} & $26 / 95$ & $27.4 \%$ & 20/95 & $21.1 \%$ & $18 / 95$ & $19.0 \%$ & $26 / 95$ & $27.4 \%$ \\
\hline & $0.31=$ & & $0.42=$ & & & & $0.55=$ & \\
\hline Area 36 & $23 / 69$ & $33.3 \%$ & $19 / 69$ & $27.5 \%$ & $9 / 69$ & $13.0 \%$ & $20 / 69$ & $29.0 \%$ \\
\hline & $0.34=$ & & $0.44=$ & & & & $0.56=$ & \\
\hline LEC & & & & & & & & \\
\hline Overall & $38 / 164$ & $23.2 \%$ & $47 / 164$ & $28.7 \%$ & $24 / 164$ & $14.6 \%$ & $43 / 164$ & $26.2 \%$ \\
\hline & $0.31=$ & & 0.43 & & & & $0.57=$ & \\
\hline Superficial & 29/107 & $27.1 \%$ & $35 / 107$ & $32.7 \%$ & 19/107 & $17.8 \%$ & 28/107 & $26.2 \%$ \\
\hline & $0.31=$ & & 0.41 & & & & $0.54=$ & \\
\hline Deep & 9/57 & $15.8 \%$ & $12 / 57$ & $21.1 \%$ & $5 / 57$ & $8.8 \%$ & $15 / 57$ & $26.3 \%$ \\
\hline & $0.31=$ & & $0.46=$ & & & & $0.61=$ & \\
\hline $\operatorname{MEC}^{b}$ & & & & & & & & \\
\hline Overall & $101 / 236$ & $42.8 \%$ & $120 / 236$ & $50.8 \%$ & $51 / 236$ & $21.6 \%$ & $101 / 236$ & $42.8 \%$ \\
\hline & 0.41 & & 0.51 & & & & 0.59 & \\
\hline Superficial & $44 / 103$ & $42.7 \%$ & $57 / 103$ & $55.3 \%$ & $27 / 103$ & $26.2 \%$ & $50 / 103$ & $48.5 \%$ \\
\hline & $0.45=$ & & $0.55=$ & & & & $0.65=$ & \\
\hline Deep & $57 / 133$ & $42.9 \%$ & $63 / 133$ & $47.7 \%$ & $24 / 133$ & $18.0 \%$ & $51 / 133$ & $38.3 \%$ \\
\hline & 0.39 & & 0.48 & & & & 0.56 & \\
\hline MEC spatial ${ }^{c}$ & & & & & & & & \\
\hline All grids & $12 / 23$ & $52.2 \%$ & $12 / 23$ & $52.2 \%$ & $3 / 23$ & $13.0 \%$ & $13 / 23$ & $56.5 \%$ \\
\hline & $0.52=$ & & $0.64=$ & & & & $0.71=$ & \\
\hline Grid only & $7 / 12$ & $58.3 \%$ & $5 / 12$ & $41.7 \%$ & $0 / 12$ & $0.0 \%$ & $6 / 12$ & $50.0 \%$ \\
\hline & $0.56=$ & & $0.66=$ & & & & $0.70=$ & \\
\hline Grid $\times$ HD & $5 / 11$ & $45.5 \%$ & $7 / 11$ & $63.6 \%$ & $3 / 11$ & $27.3 \%$ & $7 / 11$ & $63.6 \%$ \\
\hline & $0.48=$ & & $0.62=$ & & & & $0.72=$ & \\
\hline All head direction & $62 / 125$ & $49.6 \%$ & $81 / 125$ & $64.8 \%$ & $37 / 125$ & $29.6 \%$ & $73 / 125$ & $58.4 \%$ \\
\hline & $0.44=$ & & $0.55=$ & & & & $0.63=$ & \\
\hline HD only & $52 / 101$ & $51.5 \%$ & $66 / 101$ & $65.4 \%$ & 29/101 & $28.7 \%$ & $59 / 101$ & $58.4 \%$ \\
\hline & $0.43=$ & & $0.53=$ & & & & $0.62=$ & \\
\hline Border $\times$ HD & $5 / 13$ & $38.5 \%$ & $8 / 13$ & $61.5 \%$ & $5 / 13$ & $38.5 \%$ & $7 / 13$ & $61.5 \%$ \\
\hline & $0.48=$ & & $0.60=$ & & & & $0.69=$ & \\
\hline All border & $11 / 27$ & $40.7 \%$ & $14 / 27$ & $51.9 \%$ & $7 / 27$ & $25.9 \%$ & $11 / 27$ & $40.7 \%$ \\
\hline & $0.46=$ & & $0.53=$ & & & & $0.61=$ & \\
\hline Border only & $6 / 14$ & $42.9 \%$ & $6 / 14$ & $42.9 \%$ & $2 / 14$ & $14.3 \%$ & $4 / 14$ & $28.6 \%$ \\
\hline & $0.43=$ & & $0.47=$ & & & & $0.53=$ & \\
\hline Other & $26 / 85$ & $30.6 \%$ & $28 / 85$ & $32.9 \%$ & $15 / 85$ & $17.6 \%$ & $18 / 85$ & $21.1 \%$ \\
\hline & $0.35=$ & & $0.45=$ & & & & $0.54=$ & \\
\hline
\end{tabular}

${ }^{a}$ Data are mean \pm SEM. No. significant $=$ number of cells with significant coding/total number of cells tested.

${ }^{b}$ Proportions reflect MEC down-sampled data to allow direct comparison to PRC and LEC. Average SI data were not sensitive to firing rate differences and therefore were not adjusted.

Proportions reflect MEC data without down-sampling given within region comparisons only. Average SI data were not adjusted.

rized in Table 1). For example, PRC Unit 2, LEC Unit 4, and MEC Units 5 and 6 were highly selective for specific object-position conjunctions. LEC Unit 3 fired differentially during sampling of Object A relative to Object B across multiple positions. MEC grid cell 1 demonstrated particularly striking specificity for Object B in Position 1 of Context 1 (Fig. 4). Thus, even neurons typically considered dedicated to spatial representation exhibited object selective firing in animals performing this task, and vice versa.

To quantify selectivity of neurons for features of the task, we calculated an SI (Komorowski et al., 2009) for each of four task dimensions: context, position, object identity, and conjunction of object and position within a context. SI scores ranged from 0 , reflecting a lack of selectivity, to 1 , reflecting fully selective activation for one condition of a dimension. SI scores were calculated for each dimension in each recording session. We also examined whether the observed average SI value across all single units for each brain region was indicative of significant coding for each task dimension using a similar bootstrap shuffling procedure in which object and/or position identities were shuffled for each trial. Figure $5 A$ (dotted lines) reflects this bootstrap distribution. We found that each of the four task dimensions was significantly encoded in each area (all $p$ values $<0.001$; Fig. $5 A$ ). Thus, there was significant position as well as object coding in PRC, LEC, and MEC, rather than the selective position coding in MEC and selective object coding in PRC and LEC as one might expect.

We also examined selectivity between brain regions by comparing average SIs in each area for each dimension (Fig. 5A) and by comparing the fraction of neurons in each area that individually distinguished conditions of a particular dimension (Fig. 5B). MEC neurons exhibited slightly greater average selectivity relative to PRC or LEC neurons (two-way ANOVA, main effect of region, $F_{(2,2796)}=19.82, p<0.00001$; no interaction, $F_{(6,2796)}=$ $1.76, p=0.08)$. Post hoc tests confirmed that MEC exhibited significantly greater selectivity with respect to context ( $p$ values 
$<0.002)$ as well as position $(p<0.0001)$, although the magnitude of these differences is modest (Fig. 5A).

In comparing the proportions of cells in different areas that code for specific dimensions, it is important to consider that the proportion of cells significantly coding for dimensions (Fig. $5 B$ ), but not the average SI values (Fig. $5 A$ ), was sensitive to increases in firing rate. Given a higher average firing rate in MEC (PRC $2.16 \pm$ $0.19 \mathrm{~Hz}, \mathrm{LEC} 2.08 \pm 0.17 \mathrm{~Hz}$, MEC $3.07 \pm$ $0.16 \mathrm{~Hz} ; p<0.0001$ ), we randomly downsampled MEC neurons to an average firing rate equivalent to that of PRC and LEC neurons (Bonnevie et al., 2013; Mizuseki and Buzsáki, 2013). As can be seen in Figure $5 B$ and in greater detail in Table 1 , more cells in MEC significantly coded for context, position, and the conjunction of object-position information than in PRC or LEC ( $p$ values $<0.001$ ), whereas there were no differences in proportions of cells significantly coding task dimensions between PRC and LEC ( $p$ values $>0.3$ ). Thus, although analysis of single-unit properties with the SI revealed some quantitative differences in selectivity, the most striking result of this analysis is that all regions exhibit substantial coding for the full spectrum of task dimensions. This observation challenges the commonly held view that PRC and LEC selectively encode object and not spatial information, whereas MEC selectively encodes spatial information (Davachi, 2006; Eichenbaum et al., 2007).

\section{Selectivity for task dimensions is similar across anatomical subdivisions of PRC, LEC, and MEC}

When neurons were separated by laminar location of their recordings, superficial neurons in PRC exhibited greater selectivity overall relative to deep layer neurons (one-way ANOVA, $\left.F_{(1,810)}=5.22, p=0.02\right)$, but post hoc tests revealed no differences between subdivisions with respect to specific task dimensions ( $p$ values $>0.01)$. In contrast, there was no difference in stimulus selectivity between Area 35 neurons and Area 36 neurons within PRC (one-way ANOVA, $F_{(1,810)}=0.35, p=0.55$ ). There was also no difference in stimulus selectivity between superficial versus deep layers within LEC (one-way ANOVA, $F_{(1,801)}=0.64, p=$ 0.42 ). However, within MEC, superficial neurons exhibited greater selectivity than neurons in deep layers (one-way ANOVA, $\left.F_{(1,1155)}=30.16, p<0.0001\right)$. Post hoc tests confirmed that superficial layers of MEC exhibited greater object-context ( $p=$ 0.003 ) and object-position selectivity $(p=0.003)$. Again, despite some modest differences in coding across laminar subdivisions, these results reflect similar coding of task dimensions across these subdivisions.

Furthermore, contrary to the common emphasis on spatial coding in MEC grid and border cells, we found that grid cells, border cells, and head direction cells exhibited selectivity for all task dimensions, including those involving object selectivity ( $\mathrm{Ta}$ ble 1 , bottom half; all $p$ values $<0.01$ ). Indeed, the selectivity of these spatial coding cells was greater than that of the remaining MEC population (two-way ANOVA, $F_{(3,1310)}=19.62, p<$ $0.0001)$. Post hoc tests confirmed that grid cells and head direc-

\section{Lateral Entorhinal Cortex \& Perirhinal Cortex}

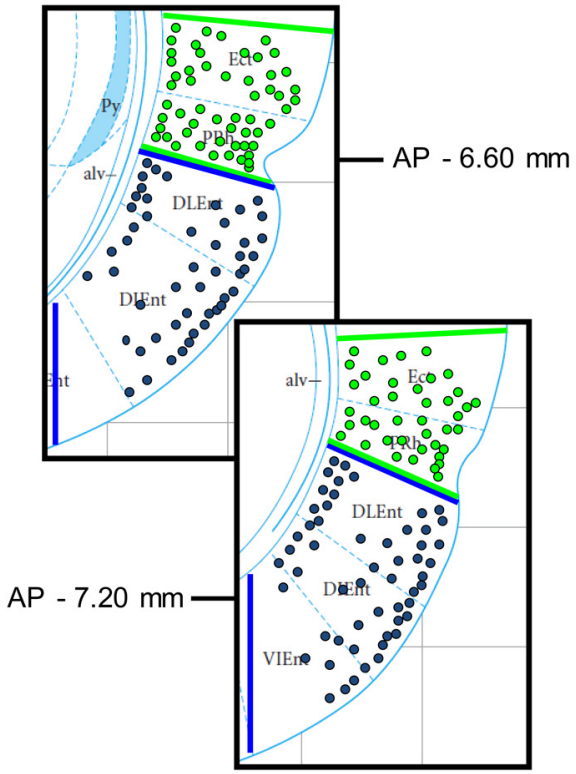

$\mathrm{ML} 4.20 \mathrm{~mm}$
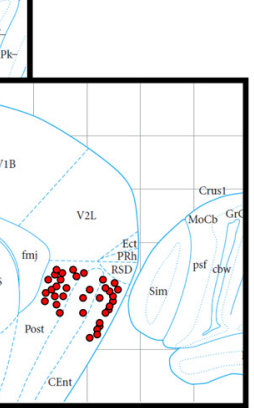

neuronal recordings in MEC. Right set of panels, Blue circles and green circles represent estimated tetrode locations in LEC and PRC, respectively. Inspection of histology for tetrode tracks and tetrode tip lesions was used in conjunction with driver turn counts and maps of tetrode locations to estimate tetrode locations during neuronal recordings.

tion cells exhibited greater selectivity for all task dimensions $(p$ values $<0.01)$ relative to the remaining MEC population, other than object coding for grid cells $(p=0.42)$ and context coding for head direction cells $(p=0.015)$. Border cells exhibited similar selectivity to the remaining MEC population ( $p$ values $>0.06$ ), and there were no differences in selectivity between the spatial cell groups ( $p$ values $>0.01$ ). Given that many grid cells and border cells also exhibit significant head direction modulation, we also compared selectivity between a combined group of grid cells and border cells without head direction modulation against that of cells with significant head direction coding only and found no differences in selectivity between these groups ( $p$ values $>0.01$; for more information, see Table 1 ). Thus, spatial selectivity properties (identified during open field foraging) may contribute in part to the identification of contexts, positions, and objects during memory performance, although all these dimensions are substantially encoded even by cells that lack the specific spatial firing properties observed during open-field foraging.

\section{Ensemble representations in PRC, MEC, and LEC organize information distinctly}

To explore how neural populations represented the task dimensions, we used an RSA. This analysis yields modest, but highly reliable, correlation coefficients that indicate that ensemble firing patterns are highly consistent between identical individual events (Fig. 5C; e.g., sampling events involving the same object in the same position and context).

First, to measure the extent to which identical events were coded similarly, population vectors for odd-numbered events were correlated against those for even-numbered events for each of the two objects within each of the two positions in each of the two contexts. The mean of those eight correlation coefficients was used to measure the ensemble similarity for identical events within each of the recording sessions ( 14 for PRC, 17 for LEC, 30 for MEC; Fig. 5C, first set of bars). For all other comparisons, 


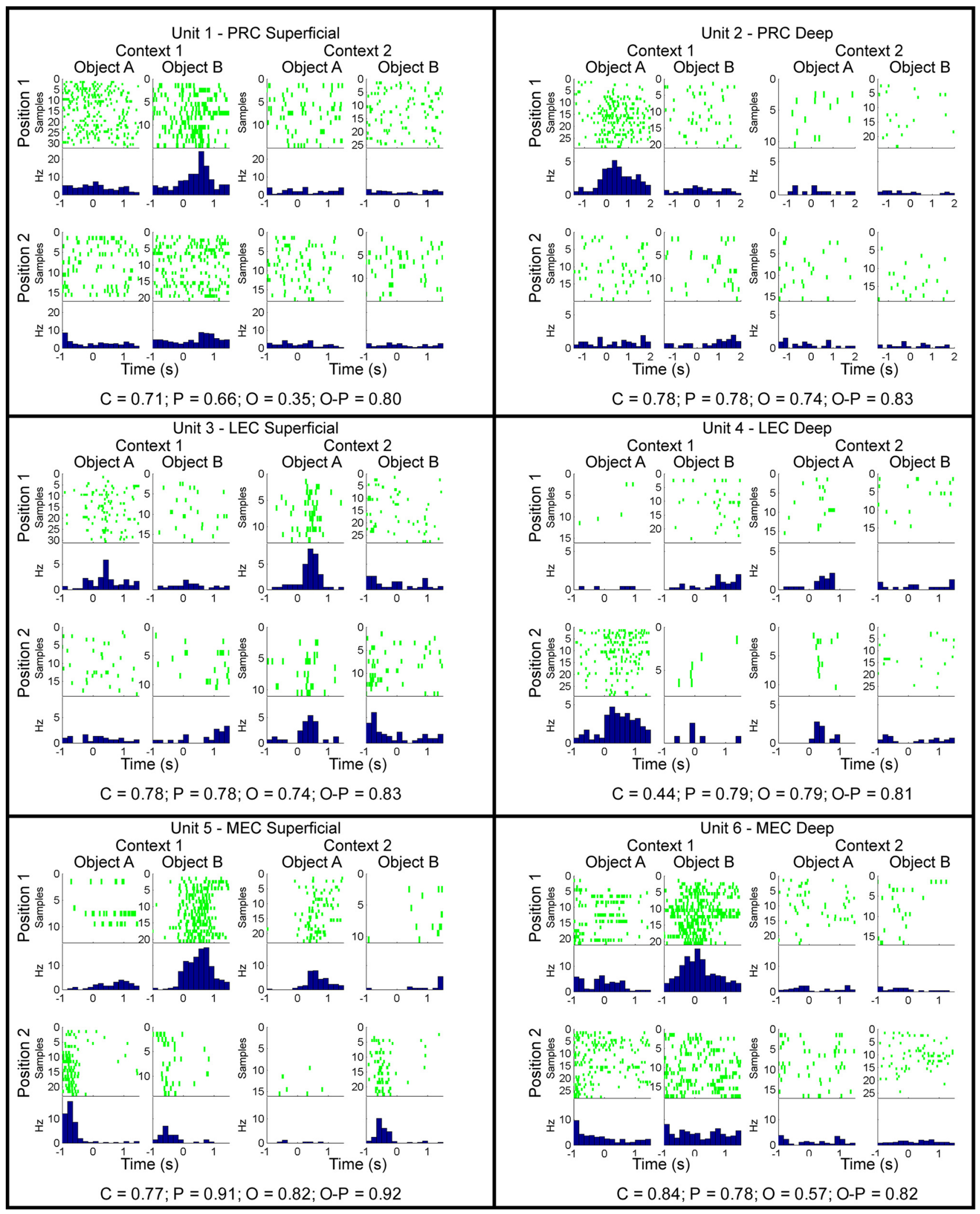

Figure 3. Examples of neuronal firing patterns during object sampling. Rasters and perievent histograms represent activity patterns during the sampling of each object (A or B) at each position (1 or 2) within each context (1 or 2). Time 0 indicates the onset of object sampling. Histograms represent firing rates in $167 \mathrm{~ms}$ time bins. Observed SI values for each example are provided at the bottom of the panel for each unit. Observed SI values (top row for each unit below) and statistical significance as determined by comparing observed SI values against bootstrapped distribution (significance level $p<0.01$ ): Unit 1: $C=0.71 ; p=0.66 ; 0=0.35 ; 0, p=0.80$. Unit 1: $C, p<0.0001 ; P, p<0.0001 ; 0, p=0.04 ; 0-P, p<0.0001$. Unit $2: C=0.78 ; p=0.78 ; 0=0.74 ; 0, p=$ 0.83. Unit $2: C, p<0.0001 ; P, p<0.0001 ; 0, p=0.0001 ; 0-P, p<0.0001$. Unit $3: C=0.50 ; p=0.37 ; 0=0.62 ; 0, p=0.73$. Unit $3: C, p=0.002 ; P, p=0.11 ; 0, p<0.0001 ; 0-P, p<0.0001$. Unit 4: $C=0.44 ; p=0.79 ; 0=0.79 ; 0, p=0.81$. Unit $4: C, p=0.03 ; P, p<0.0001 ; 0, p<0.0001 ; 0-P, p=0.0002$. Unit $5: C=0.77 ; p=0.91 ; 0=0.82 ; 0, p=0.92$. Unit $5: C, p=0.0001$; $P, p<0.0001 ; 0, p<0.0001 ; 0-P, p<0.0001$. Unit $6: C=0.84 ; p=0.78 ; 0=0.57 ; 0, p=0.82$. Unit $6: C, p<0.0001 ; P, p<0.0001 ; 0, p=0.0004 ; 0-P, p<0.0001$. C, Context; $0-C$, object-context; 0 , object; $0-P$, object-position; $P$, position. 


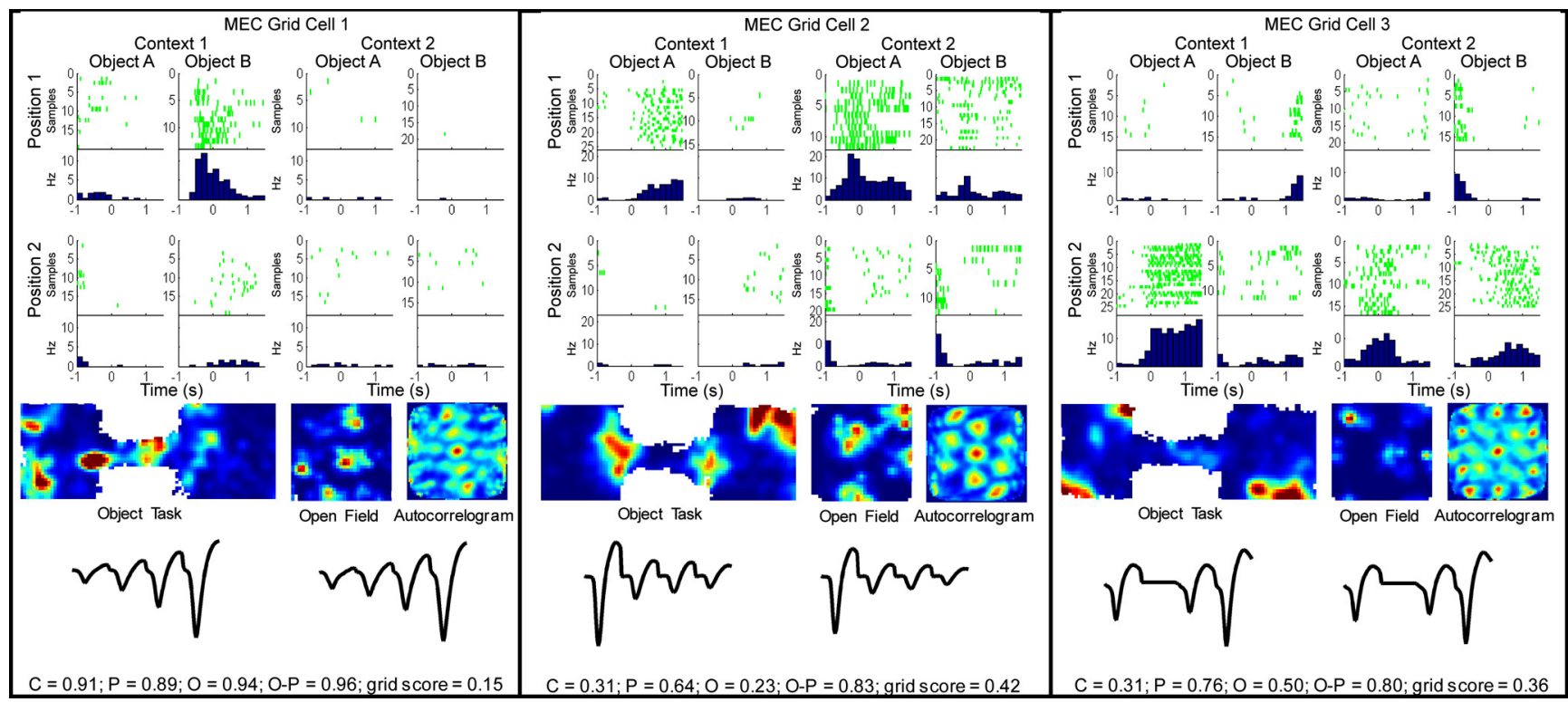

Figure 4. Examples of neuronal firing patterns for grid cells during object sampling. Rasters and perievent histograms (top half) for grid cells as in Figure 3 . In the bottom half of each example, from left to right, firing rate maps for the entire session of the behavioral task, the open field session, and an autocorrelogram for the open field session. Below that are the average tetrode waveforms for spikes isolated during the behavioral (left) and open field (right) sessions, respectively. Observed SI values are plotted at the bottom of the panel for each unit. Observed SI values (top row for each unit below) and statistical significance as determined by comparing observed SI values against bootstrapped distribution (significance level $p<0.01$ ): Grid cell 1:C $=0.91 ; p=0.89 ; 0=0.94 ; 0$, $p=0.96$; grid score $=0.15$. Grid cell $1: C, p=0.0004 ; \mathrm{P}, p=0.0003 ; 0, p<0.0001 ; 0-\mathrm{P}, p<0.0001$. Grid cell $2: \mathrm{C}=0.31 ; p=0.64 ; 0=0.23 ; 0, p=0.83 ;$ grid score $=0.42$. Grid cell 2:C, $p=$ $0.14 ; \mathrm{P}, p=0.0001 ; 0, p=0.29 ; 0-\mathrm{P}, p<0.0001$. Grid cell $3: \mathrm{C}=0.31 ; p=0.76 ; 0=0.50 ; 0, p=0.80 ;$ grid score $=0.36$. Grid cell $3: C, p=0.14 ; \mathrm{P}, p<0.0001 ; 0, p=0.007 ; 0-\mathrm{P}, p<0.0001$. C, Context; 0 -C, object-context; 0 , object; $0-P$, object-position; P, position.

population vectors for object-sampling events of each type were correlated with those for a different type of event. To measure the similarity of ensemble representations for different objects sampled at the same position, population vectors for odd-numbered events for one object were correlated against even-numbered events for the other object, and vice versa (even-numbered events for the first object against odd-numbered events for the second object), to compose eight total correlations, and the mean of those correlation coefficients was used to measure the representational similarity for different objects (holding position and context constant) in each session (Fig. 5C, second set of bars). The same approach was used to measure the representational similarities for the same or different objects at different positions within a context, including the separation of odd- and evennumbered events to ensure that similar amounts of data were used in all analyses. To assess the similarity of ensemble representations for the same object between positions, population vectors for sampling events involving an object in one position were correlated with those for the same object in the other position within the same context (Fig. $5 C$, third set of bars) or with that for the other object in the other position within the same context (Fig. $5 C$, fourth set of bars), again comparing odd-numbered against even-numbered events and vice versa. To assess the similarity of ensemble representations of objects between contexts, population vectors for odd-numbered and even-numbered events for the same object (Fig. $5 C$, fifth set of bars) or different objects (Fig. $5 C$, sixth set of bars) at positions between contexts were similarly correlated. The mean correlation coefficients for individual rats for all 3 brain regions are presented in Figure 6, showing that the overall pattern of correlations was similar across individual subjects.

Although a statistical comparison of the individual bars in Figure $5 C$ is not easily interpreted, it is clear that PRC and LEC share a highly similar pattern of correlations for events varying across dimensions; and on some dimensions, these are distinct from that found in MEC. In particular, whereas MEC ensembles are similar for different objects in the same position $(p<0.0001)$, PRC and LEC ensembles have independent representations for different objects in the same position (correlation not significantly different from $0, p$ values $>0.3$ ). Conversely, whereas PRC and LEC ensembles have similar representations for the same object at different positions ( $p$ values $=0.002$ ), MEC ensembles do not $(p>0.5)$. This dissociation reflects stronger position coding in MEC ensembles and stronger coding of object information in PRC and LEC. It is also notable that, in all areas, representations of different objects in different positions and comparisons between contexts were negatively correlated (all $p$ values $<0.005)$, indicating strong pattern separation of representations across these dimensions.

To better quantify these distinct patterns of activity, these correlations were combined in several ways described below to provide a straightforward measure of the similarity of population representations associated with each task dimension. We used a $\mathrm{d}^{\prime}$ metric (as in McKenzie et al., 2014) to measure the separation of the distributions of the correlation coefficients for specific task features from 0 or against the distribution of coefficients from an appropriately opposing condition (for each comparison, see Materials and Methods). As seen in Figure 5D, analysis of the ensemble representations revealed both similarities and differences in the representation of task dimensions. A two-way ANOVA on the $\mathrm{d}^{\prime}$ metric indicated a main effect of task dimension $\left(F_{(3,232)}=\right.$ $23.75, p<0.00001)$, a main effect of region $\left(F_{(2,232)}=3.57, p=\right.$ $0.03)$, and a significant interaction $\left(F_{(6,232)}=7.21, p<0.00001\right)$. Post hoc tests indicated that PRC, LEC, and MEC populations equally strongly represented contexts ( $p$ values $>0.4$ ), whereas MEC exhibited stronger representation of position information than PRC ( $p=0.001)$ and LEC ( $p=0.001)$. In contrast, PRC and LEC exhibited stronger representation of object information ( $p$ 
A

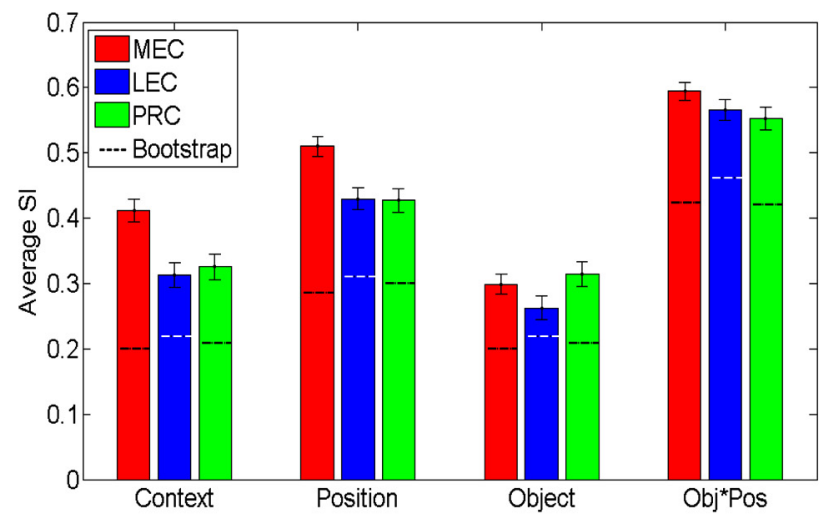

C

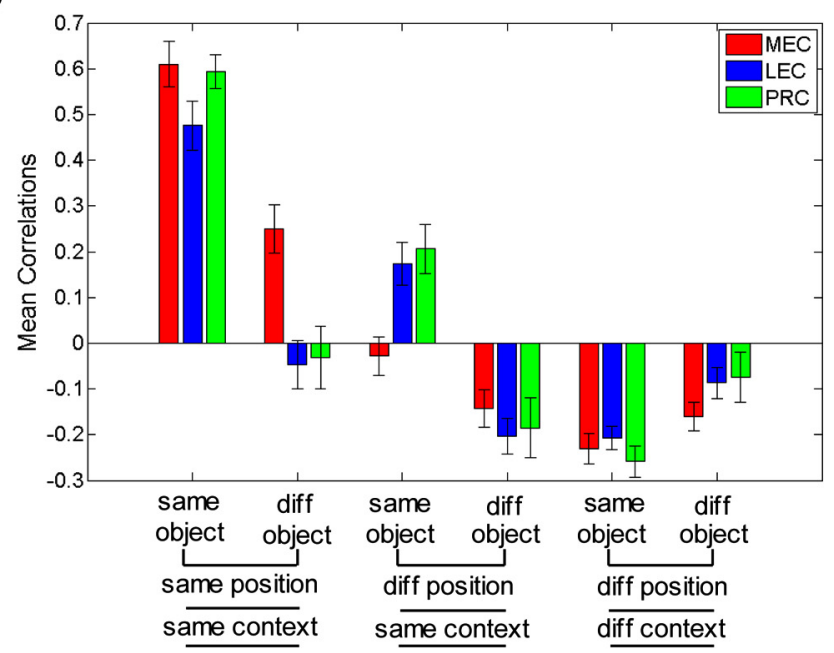

B

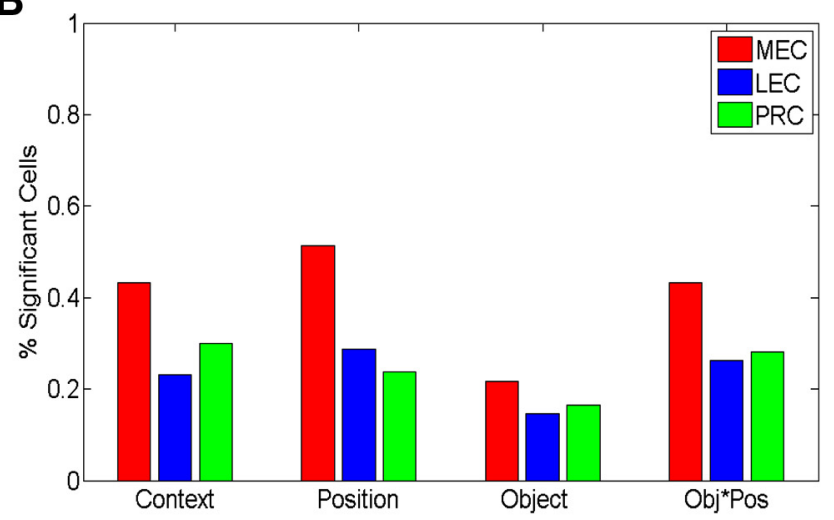

D

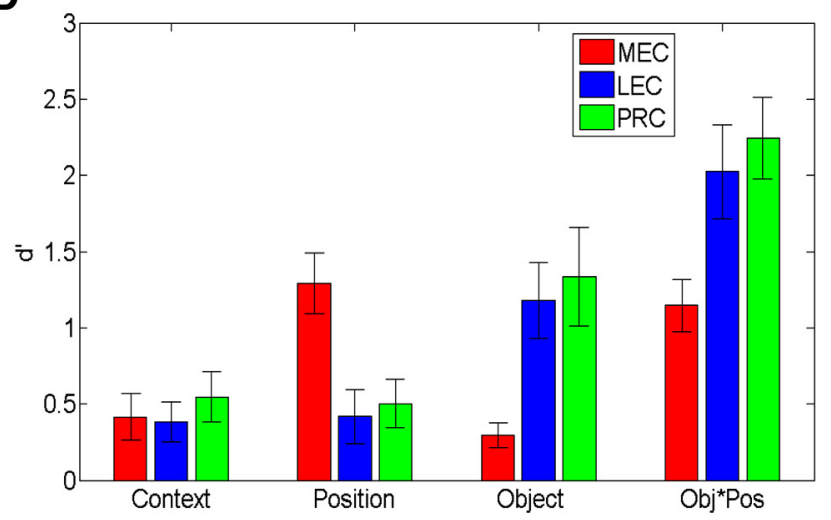

Figure 5. Single-unit and population coding for task dimensions. A, Average SI \pm SE for task dimensions during object sampling in PRC, LEC, and MEC. Dotted lines indicate the average SI values from a bootstrapped distribution in which the position and/or object identities were shuffled for each trial (preserving firing rates and patterns). Even with this stringent criterion to determine coding of task dimensions, we found significant coding for all dimensions in all three regions. $\boldsymbol{B}$, Percentage of cells that significantly code for task dimensions after controlling for firing rate differences. $\boldsymbol{C}$, Mean correlations coefficients \pm SE for comparisons between object sampling events that are the same or different in distinct dimensions (object, position, context). Diff, Different. $\boldsymbol{D}$, Strength of population coding of different task dimensions, measured as $\mathrm{d}^{\prime}$ distance between distributions of z-scored firing rates for context, position coding, object coding, and objects in specific positions (Obj*Pos).

values $=0.0001)$ and object-position conjunction information than MEC (PRC vs MEC, $p=0.009$; PRC vs MEC, $p=0.001$ ). Consistent with findings of mixed selectivity from the SI analysis, analysis of ensemble representations in PRC, LEC, and MEC indicated that all regions encode all task dimensions (all $p$ values $<0.01$ ), but the observed double dissociation between MEC representation of position and PRC-LEC representation of objects and object-position conjunctions indicates a difference in the underlying organization of representations in PRC-LEC and MEC.

To test the generality of the RSA approach, ensembles of recorded neurons were also analyzed using the cosine separation of population vectors to characterize the similarity of representations. Strength of coding dimensions to different task features were identified by comparing $\mathrm{d}^{\prime}$ distributions of mean cosine scores similar to the RSA approach. Analysis of these d' comparisons with a two-way ANOVA revealed a very similar pattern of population coding as with the RSA approach (Fig. $7 A$ ), indicating a main effect of task dimension $\left(F_{(3,232)}=19.86, p<0.00001\right)$, no main effect of region $\left(F_{(2,232)}=0.18, p=0.84\right)$, and a significant interaction $\left(F_{(6,232)}=6.42, p<0.00001\right)$. Similar to the RSA results, post hoc tests indicated that PRC, LEC, and MEC popula- tions equally strongly represented contexts ( $p$ values $>0.3$ ) while also indicating similar representation of object-position conjunctions $(p$ values $>0.2$ ). In contrast to those similarities in function, MEC exhibited stronger representation of position information than PRC $(p=0.006)$ and $\operatorname{LEC}(p=0.001)$, whereas PRC and LEC exhibited stronger representation of object information (PRC vs MEC, $p=0.01$; PRC vs MEC, $p=0.0005$ ). Despite reflecting more similar representations with respect to object-position conjunctions, these results are nonetheless highly consistent with those found with the RSA approach, and they suggest a distinct, but complementary, organization of object and spatial information at the population level between PRC-LEC and MEC.

We further tested the generality of these findings across analytical approaches by estimating the probability that a pattern of ensemble firing rates was recorded in each of 8 object in position combinations using a Bayesian decoding algorithm. Because there is a probability associated with each object/position combination, we could determine whether there were different hierarchies of coding probabilities among the areas examined. MEC ensembles exhibited nearly equivalent coding probabilities for position and object/position conjunctions, followed by object 

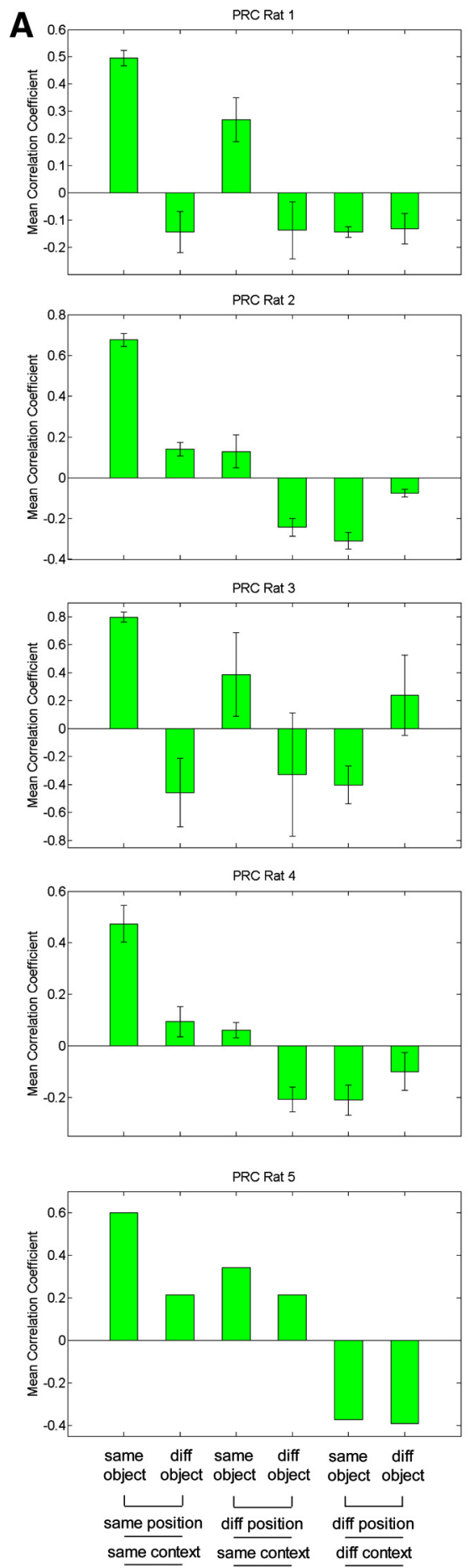
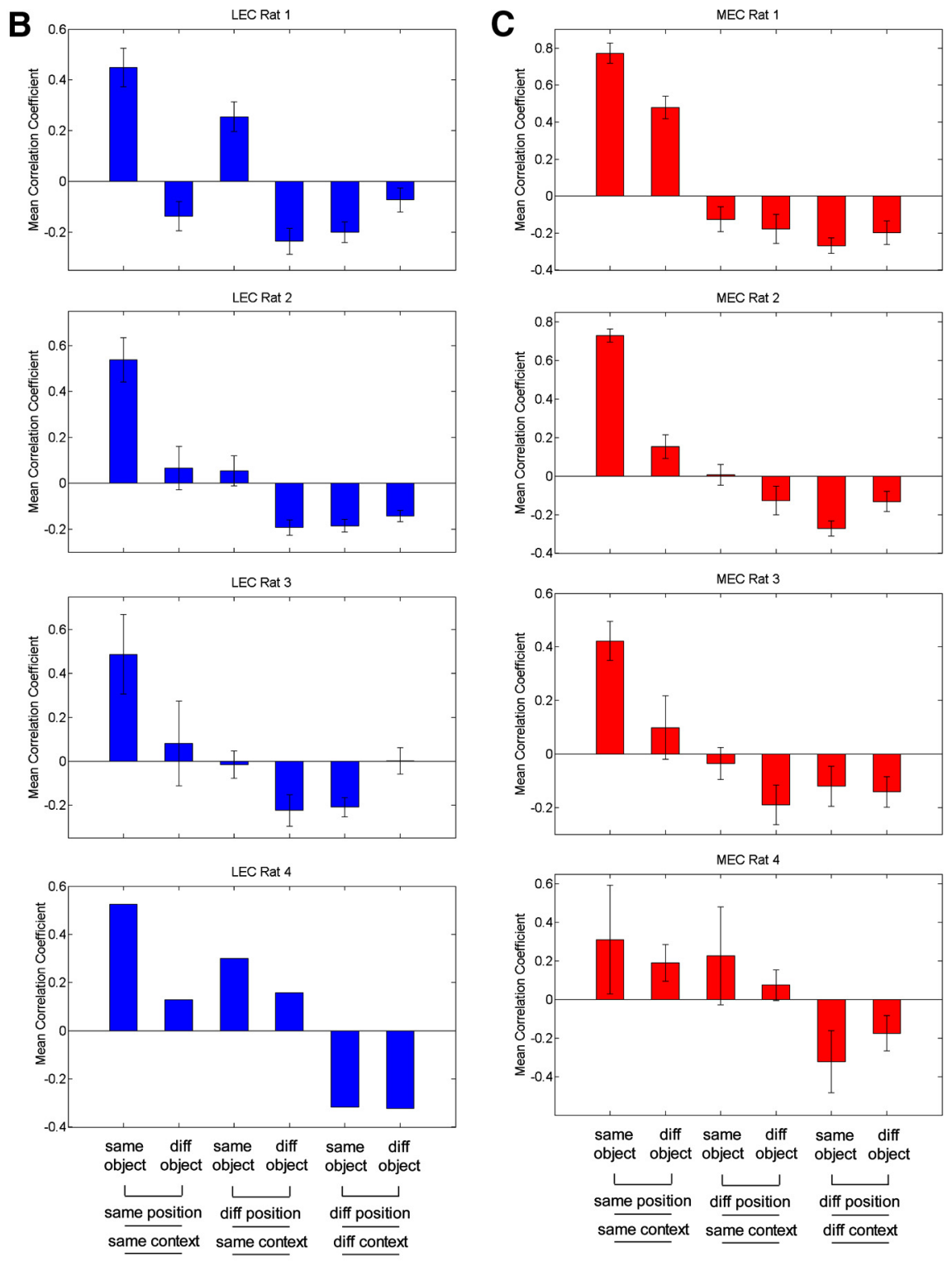

Figure 6. Mean correlation coefficients for individual rats. Mean correlation coefficients for comparisons between object sampling events that are the same or different in distinct dimensions (object, position, context) for individual subjects in (A) PRC, (B) LEC, and (C) MEC. diff, Different.

and context coding probabilities. In contrast, LEC ensembles exhibited object/position and object coding probabilities most strongly, followed by context coding and then position coding probability. In PRC, the hierarchy was less distinct with strong object/position coding, followed by relatively equivalent coding of context, position, and object information. With respect to the decoding probabilities for task dimensions, for all regions ensembles were most likely to have originated from trials with the same object and position combination (mean probability $\mathrm{PRC}=0.29$; $\mathrm{LEC}=0.32 ; \mathrm{MEC}=0.42$ ), which was greater than the probability of the neural activity originating from trials with different objects or positions (PRC mean probability $=0.15, \mathrm{~d}^{\prime}=0.66, p<0.01$; LEC mean probability $=0.15, \mathrm{~d}^{\prime}=0.71, p<0.001$; MEC mean probability $\left.=0.13, \mathrm{~d}^{\prime}=0.81, p<0.001\right)$. These differences in probabilities reflect strong object-in-position coding. PRC and LEC exhibited the greatest object coding, followed by MEC, indicated by a greater probability of ensembles originating from sampling events of the same object in different positions (PRC mean probability $=0.18$; LEC mean probability $=0.19 ; \mathrm{MEC}=$ 0.11 ) than different objects in different positions within a context 


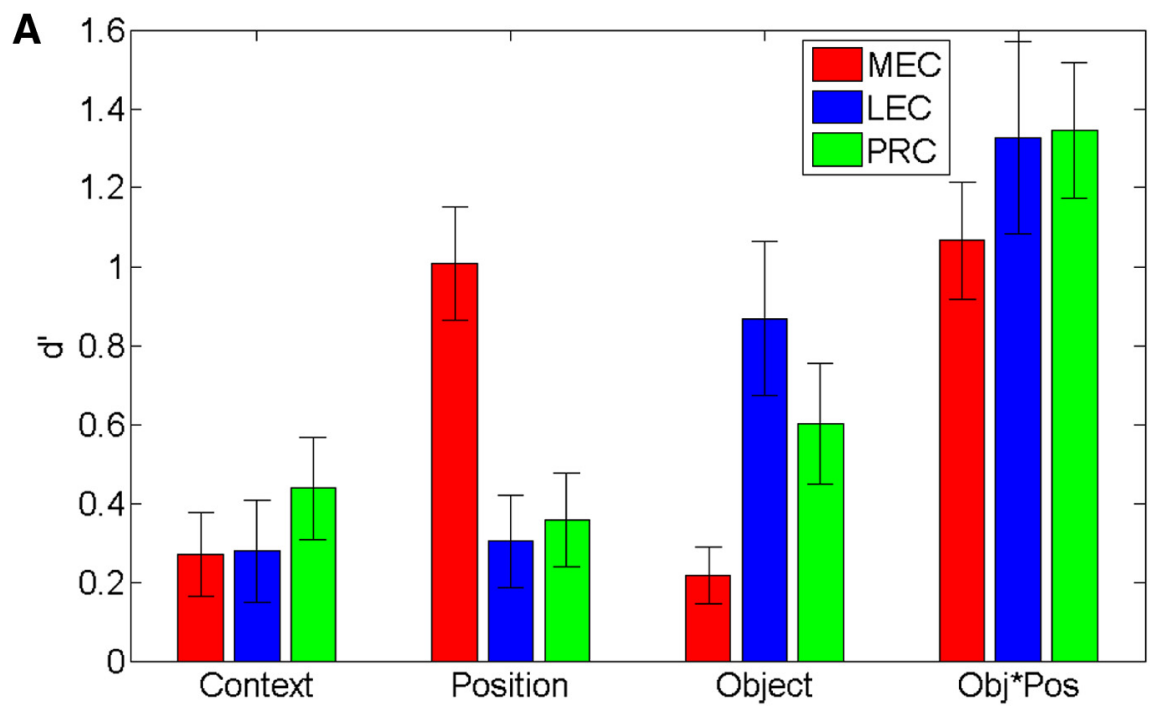

B

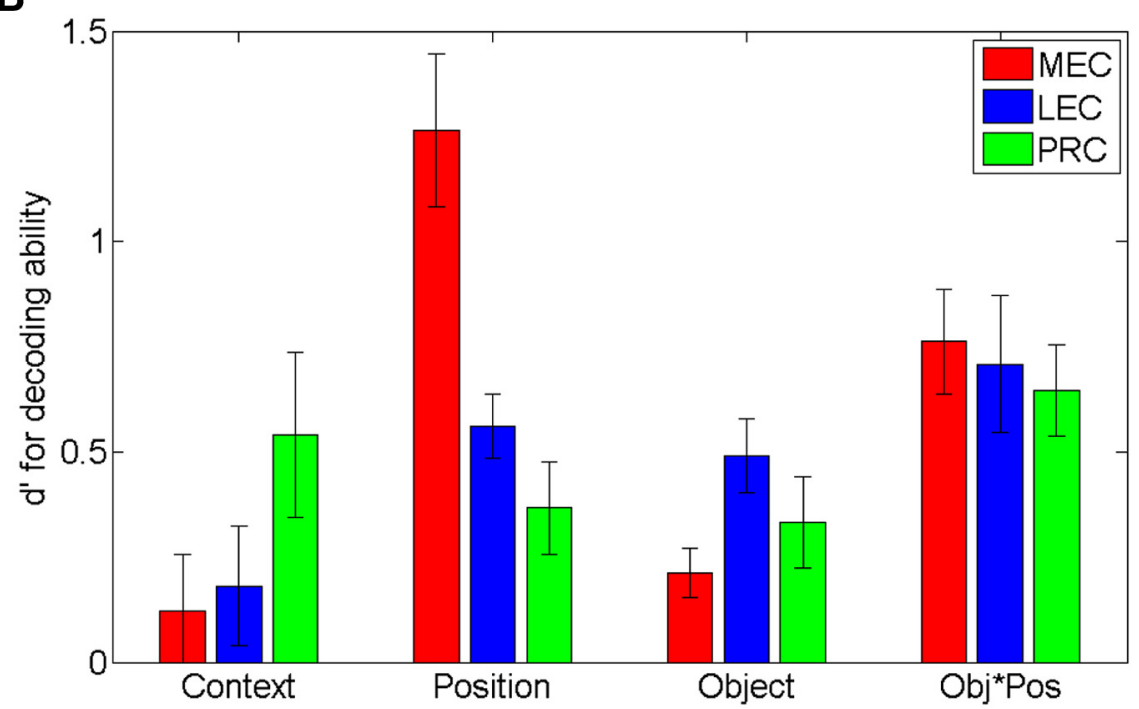

Figure 7. Population coding of task dimensions in MEC and LEC during object sampling. $\boldsymbol{A}$, Strength of coding as in Figure 5D, except using $\mathrm{d}^{\prime}$ distance between distributions of average cosine values for firing rates normalized by the maximum firing rate during object sampling. $\boldsymbol{B}$, Strength of coding using $\mathrm{d}^{\prime}$ distance between mean probabilities that patterns of ensemble firing rates were recorded in each of the eight object in position combinations determined by a Bayesian decoding algorithm.

To directly compare the findings among brain regions and relate these findings to the correlation and cosine separation analyses, we compared the decoding probabilities for task dimensions using the $\mathrm{d}^{\prime}$ metric (Fig. 7B). An ANOVA was conducted on the $\mathrm{d}^{\prime}$ values generated with the Bayesian decoding algorithm. This analysis indicated no main effect of region $\left(F_{(2,168)}=1.04, p=0.35\right)$ but did indicate a significant interaction $\left(F_{(3,168)}=5.47\right.$, $p<0.0001)$. Post hoc tests revealed an underlying pattern that was highly similar to those found in the ensemble correlation and cosine separation analyses. LEC exhibited greater object coding than MEC $(p=0.005)$, and MEC exhibited greater position coding than PRC or LEC ( $p$ values $<0.001$ ). However, PRC did not exhibit greater object coding than MEC $(p>0.05)$, potentially because of a higher probability of context decoding in PRC. In contrast, there were no differences between PRC, LEC, and MEC with respect to context or object-position conjunctive coding as determined by the decoding algorithm ( $p$ values $>0.05$ ). Together, this group of analyses demonstrates reliable patterns of activity in PRC, LEC, and MEC, suggesting distinct but complementary roles in processing object and spatial information.

\section{Coding of task dimensions across time during object sampling}

We also compared these brain areas with respect to the temporal dynamics of information coding among the different task dimensions based on the correlation RSA (as in Fig. 5D). Significant sustained position coding was evident in MEC earliest, followed $\sim 1-2$ s later by PRC and LEC (Fig. 8A). Furthermore, position coding appeared to peak around the onset of object sampling, which was particularly pro-

$\left(\right.$ PRC mean probability $=0.10, \mathrm{~d}^{\prime}=0.34, p<0.001$; LEC mean probability $=0.07, \mathrm{~d}^{\prime}=0.42, p<0.001$; MEC mean probability $\left.=0.07, \mathrm{~d}^{\prime}=0.24, p<0.001\right)$. In contrast, MEC exhibited the greatest position coding, followed by PRC and LEC, reflected by a greater probability of ensembles originating from sampling events in the same position (PRC mean probability $=0.20$; LEC mean probability $=0.22 ; \mathrm{MEC}=0.28)$ than sampling events at different positions within a context (PRC mean probability $=$ $0.14, \mathrm{~d}^{\prime}=0.32, p<0.001$; LEC mean probability $=0.13, \mathrm{~d}^{\prime}=$ $0.53, p<0.001$; MEC mean probability $=0.09, \mathrm{~d}^{\prime}=1.29$, $p<0.001)$. Finally, a greater probability of ensembles originated from sampling events within contexts (PRC mean probability $=0.17 ;$ LEC mean probability $=0.17 ; \mathrm{MEC}=0.19)$ than across contexts (PRC mean probability $=0.08 ; \mathrm{d}^{\prime}=0.59$, $p<0.001$; LEC mean probability $=0.09, \mathrm{~d}^{\prime}=0.22, p<0.001$; MEC mean probability $=0.07, \mathrm{~d}^{\prime}=0.09, p=0.05$, not significant), indicating approximately equivalent context coding in LEC and MEC. nounced in MEC $\left(\right.$ PRC peak $=-400 \mathrm{~ms}, \mathrm{~d}^{\prime}=0.35, p<0.001$; LEC peak $=-400 \mathrm{~ms}, \mathrm{~d}^{\prime}=0.30, p<0.001$; MEC peak $=0 \mathrm{~ms}$, $\left.\mathrm{d}^{\prime}=0.82, p<0.001\right)$. In contrast, significant object coding was evident in PRC and LEC at the onset of object sampling (Fig. 8B) with sustained peak firing beginning at $\sim 500 \mathrm{~ms}$ after the onset of object sampling $\left(\right.$ PRC peak $=800 \mathrm{~ms}, \mathrm{~d}^{\prime}=0.32, p<0.001$; LEC peak $\left.=1200 \mathrm{~ms}, \mathrm{~d}^{\prime}=0.31, p<0.001\right)$, whereas MEC did not exhibit significant object coding in this analysis (MEC peak = $\left.600 \mathrm{~ms}, \mathrm{~d}^{\prime}=0.07, p=0.24\right)$. Despite these differences in position and object coding, there was less difference in the onset of objectin-position coding among PRC, LEC, and MEC (Fig. 8C). Sustained, significant coding for this task dimension was evident at or just after the onset of object sampling $(0 \mathrm{~ms})$ with peak firing at $\sim 1-1.5 \mathrm{~s}$ after the onset of object sampling (PRC peak $=1200$ $\mathrm{ms}, \mathrm{d}^{\prime}=0.82, p<0.001 ;$ LEC peak $=1000 \mathrm{~ms}, \mathrm{~d}^{\prime}=0.74, p<$ 0.001 ; MEC peak $\left.=1400 \mathrm{~ms}, \mathrm{~d}^{\prime}=0.71, p<0.001\right)$. It should also be noted that the timing pattern is unique for each of these di- 
mensions, reflecting distinct coding of each of these features of events. Collectively, the temporal dynamics of coding of different task dimensions further distinguishes MEC as emphasizing position coding, whereas PRC and LEC population activity reflects earlier and stronger object coding, consistent with the results of the other population analyses.

\section{PRC-LEC and MEC exhibit distinct hierarchical organizations of information}

To explore the organization of dimensions represented in PRC, LEC, and MEC populations, we performed an additional analysis in which similarities between population vectors for each sample type were iteratively clustered according to their mean correlation coefficients. The highest correlations result in the tightest clustering of nodes in the organizational structure and successively lower correlations reflect greater separation of representations, resulting in a systematic hierarchical organization of task dimensions (Fig. 9). At the top of the hierarchy for all regions, representations were strongly separated (negatively correlated) by events that occurred in different contexts. Within each context-based cluster, in PRC and LEC, events within each context were next separated by the object presented. Then, within each object cluster, the positions where objects occurred were separated. In MEC, events within each context were also strongly separated (negatively correlated). Then, in contrast to PRC and LEC, within each context-based cluster, events were separated by the positions in which objects were sampled. Then within each position representation, objects were separated. Finally, in all areas, representations of identical events are most closely associated. Thus, although PRC, LEC, and MEC exhibit great similarity in mixed selectivity at the single-neuron level, RSA revealed an emergent distinction between these areas in complementary organizations of event representations.

\section{Discussion}

The present observations on PRC, LEC, and MEC neuronal firing patterns quite dramatically reject the notion that PRC and LEC neurons selectively encode object information, whereas MEC neurons selectively encode spatial information. Given the direct and indirect connections between these areas (Witter et al., 1989; Suzuki and Amaral, 1994; Burwell and
A
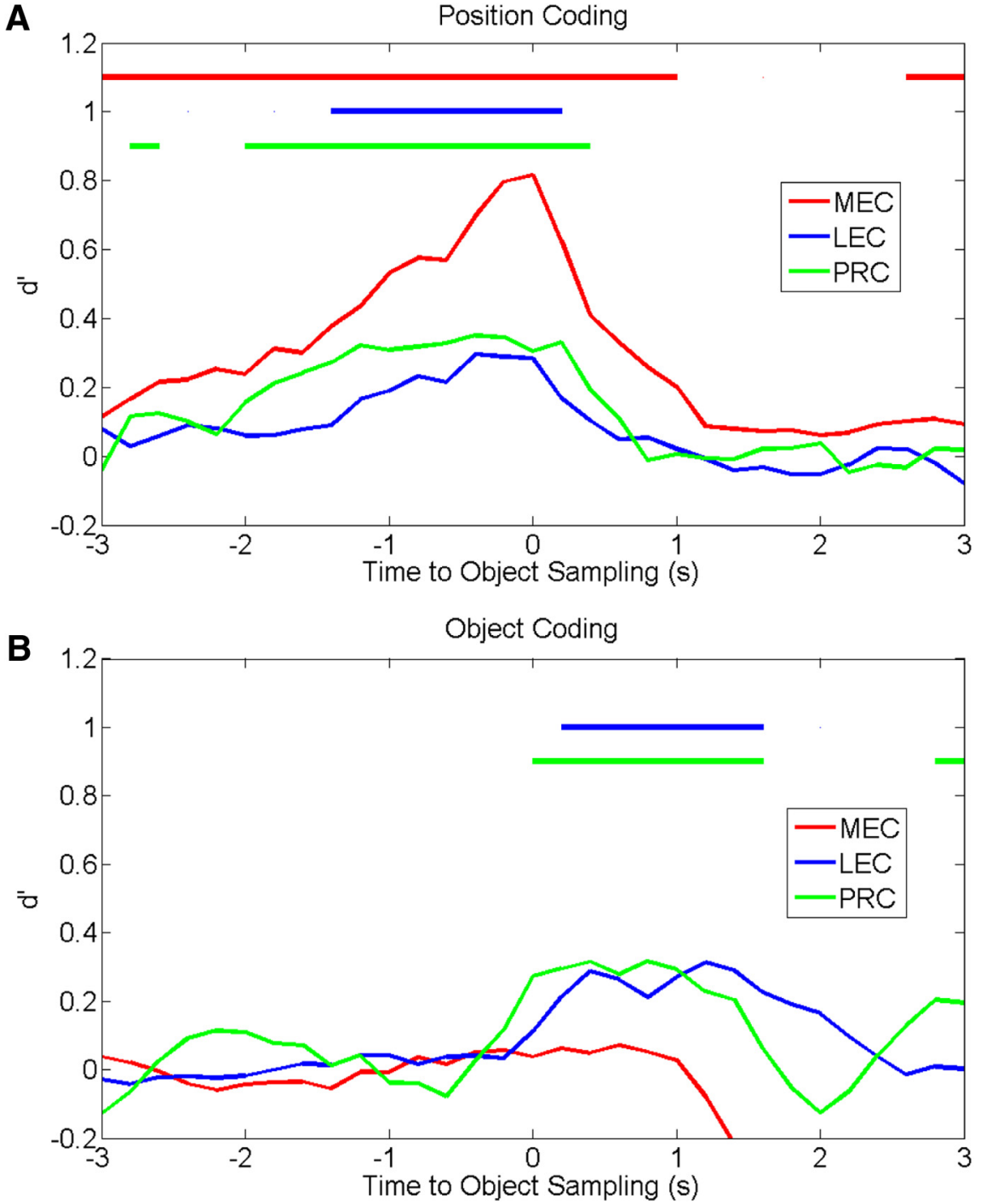

C

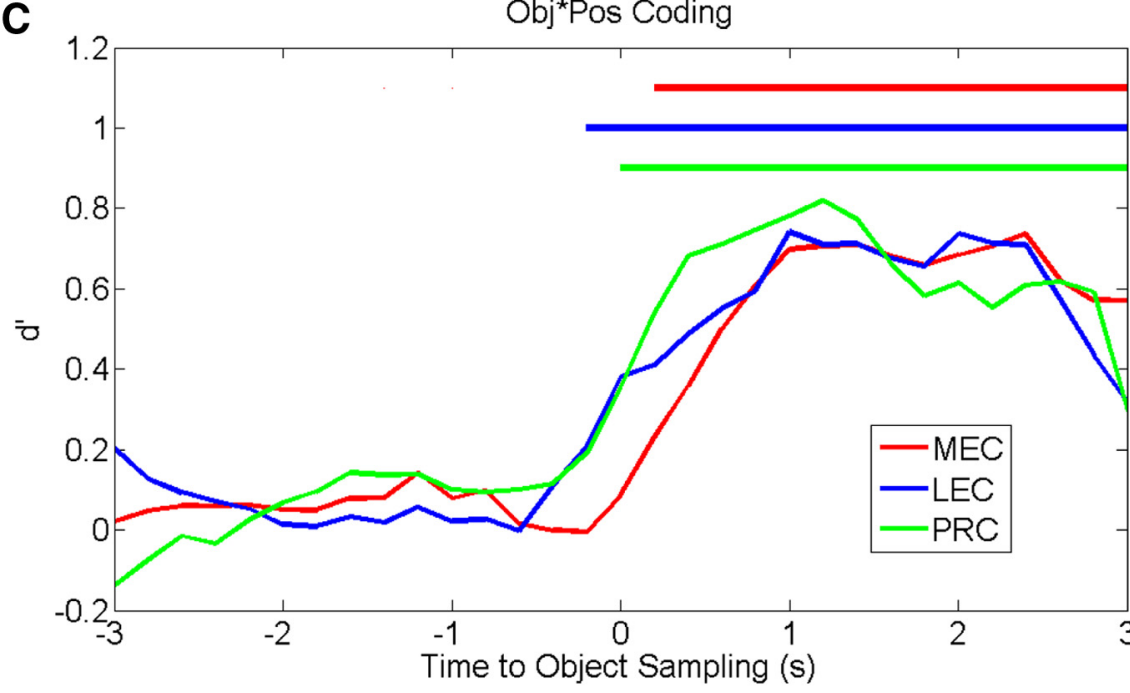

Figure 8. Coding for task dimensions is expressed at different times across regions during object sampling. The average $\mathrm{d}^{\prime}$ strength calculated from mean correlations (as in Fig. 5D) for each task dimension is depicted over time (using $200 \mathrm{~ms}$ bins) before and after the onset of object sampling ( $0 \mathrm{~s}$ ). MEC exhibits the earliest and strongest position coding compared with the pattern for $\operatorname{PRC}$ or LEC $(\boldsymbol{A})$, whereas PRC and LEC exhibit early and strong object coding, which is not observed in $\operatorname{MEC}(\boldsymbol{B})$, and all three regions exhibit a similar degree of conjoint object-position coding that peaks later than separate coding of positions and objects ( $\boldsymbol{C}$. Color-coded bars at the top of each graph represent periods in which the dimension was significantly coded. 

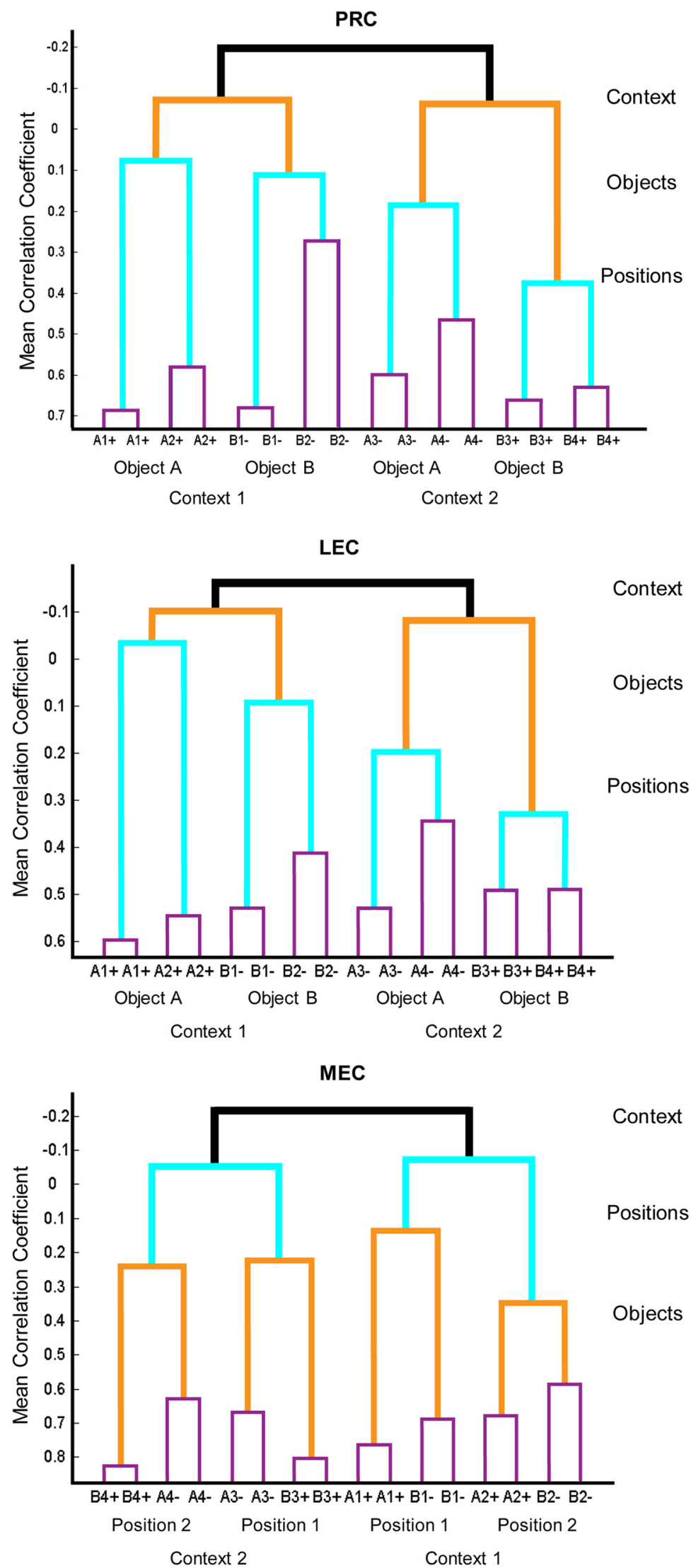

Figure 9. Hierarchical organization of event representations by task dimensions. Dendrograms illustrating organization of population representations in PRC, LEC, and MEC during object sampling for each specific type of object sampling event (a particular object in a specific position within one context). Letters indicate 0bjects A or B in Positions 1 or 2 (Context 1) or Positions 3 or 4 (Context 2) with + or - indicating whether the stimulus was reinforced. Repeated events (e.g., $A 1+$ and $A 1+$ ) indicate
Amaral, 1998; Kerr et al., 2007), it should be expected that information processed in each of these areas is shared with the other. However, the extent to which individual PRC, LEC, and MEC neurons, including even grid cells, border, and head direction cells, process both object and spatial information equally is surprising. At the same time, population analyses strikingly dissociated PRC and LEC from MEC networks as prioritizing object and spatial information, respectively. These observations reinforce conclusions from recent studies that the full content and organization of representations in higherorder brain areas are most clearly revealed in population analyses (Rigotti et al., 2013; Shamir, 2014).

Given the previous reports of striking coding of specific spatial dimensions (position, direction, borders) in MEC neurons, it was quite surprising that MEC neurons of all subtypes (grid, border, head direction, other) strongly encoded object information and did so at least as well as PRC and LEC neurons. Also, given previous observations suggesting a lack of spatial coding by PRC and LEC neurons (Hargreaves et al., 2005; Deshmukh et al., 2012), it was quite surprising that PRC and LEC neurons strongly encoded position and context, although not quite matching that seen in MEC. The most obvious difference in the present study and previous work is that both spatial and object dimensions were salient features of the task demands in this context-guided object-reward association paradigm. It is important to consider that spatial context, which determined the reward associations and therefore was critical for task performance, and position within contexts, which was not a relevant dimension for task performance, were both strongly encoded by PRC, LEC, and MEC. Perhaps, once contextual spatial cues are relevant, both global and local spatial information becomes sufficiently salient to demand representation. This interpretation is consistent with other recent studies that have previously identified spatial coding in a minority of LEC cells (Deshmukh et al., 2011; Tsao et al., 2013). Both studies provided evidence of spatial "memory" fields at prior locations when objects were

$\leftarrow$

comparison of population vectors for even- and oddnumbered events for that event type (see Online Methods). Black represents correlation of population vectors between contexts. Red represents correlations between objects. Green represents correlations between positions. Blue represents correlations between even- and odd-numbered trials. 
moved in or removed from the environment, whereas Deshmukh et al. (2011) also provided examples of activity similar to place field firing when objects were presented. In addition, studies on earlier stages of information processing before LEC and MEC (i.e., in the PRC and postrhinal cortex) have also reported mixed selectivity of neuronal activity in animals performing memory tasks where objects and spatial choices are relevant (Furtak et al., 2012; Ahn and Lee, 2015). The present findings suggest that both LEC and MEC also represent organizations of objects and events within spatial contexts (see also Knierim et al., 2013) and that even PRC exhibits a combination of object and spatial coding.

It was particularly striking that grid cells strongly encoded object information, often differentiating objects sampled at the same location (Fig. 4). In contrast to the common view that grid cells are specialized for position coding, results from the present study strongly suggest that grid cells possess strong object-coding properties that have been largely overlooked to this point. Future investigation of these properties could yield further insights about the organization of information in cortical structures of the hippocampal region and beyond, given the detailed understanding of grid cell function and organization (Moser and Moser, 2013; Moser et al., 2013; Witter et al., 2014).

The RSA used here revealed that, within equivalent coding of specific dimensions by single PRC, LEC, and MEC neurons, populations of neurons in all these areas developed distinct systematic organizations of those dimensions. Notably, both areas developed quite separate (anticorrelated) networks consistent with the opposite reward associations of objects in the two contexts. Other recent studies have shown that grid cell firing patterns in MEC are responsive to contextual differences (Marozzi et al., 2015) as well as a merging of spatial contexts (Carpenter et al., 2015). Furthermore, strong pattern separation of contextual representation was also observed in the hippocampus in a previous study that used the same task (McKenzie et al., 2014). Thus, contrary to other work suggesting that pattern separation occurs selectively within subregions of the hippocampus (Lee et al., 2004; Leutgeb et al., 2004; Bakker et al., 2008), here the entire hippocampal system responds to a strong demand for reducing interference between the opposite object associations in the two contexts by robust pattern separation.

Within the two context-based networks, PRC, LEC, and MEC organize the object and spatial information quite differently. Within each context-based network, the closely interconnected PRC and LEC populations distinguish objects, and then only within each object representation distinguish the positions where objects are sampled. Conversely, within each context-based network, MEC populations distinguish the positions where object sampling occurs, and then only within each position representation distinguish the objects at those locations. Notably, the population representation within the MEC is very similar to that observed in the hippocampus (McKenzie et al., 2014), suggesting a prominent role of MEC in driving spatial organization in the hippocampus. Overall, this pattern of findings suggests we move from thinking about modality-specific processing areas within the hippocampal system (Eichenbaum et al., 2007) to conceiving the hippocampal system as composed of interconnected areas, each of which processes all the information but differently organizes the dimensions of information processing.

\section{References}

Ahn JR, Lee I (2015) Neural correlates of object-associated choice behavior in the perirhinal cortex of rats. J Neurosci 35:1692-1705. CrossRef Medline
Bakker A, Kirwan CB, Miller M, Stark CE (2008) Pattern separation in the human hippocampal CA3 and dentate gyrus. Science 319:1640-1642. CrossRef Medline

Bonnevie T, Dunn B, Fyhn M, Hafting T, Derdikman D, Kubie JL, Roudi Y, Moser EI, Moser MB (2013) Grid cells require excitatory drive from the hippocampus. Nat Neurosci 16:309-317. CrossRef Medline

Brandon MP, Bogaard AR, Libby CP, Connerney MA, Gupta K, Hasselmo ME (2011) Reduction of theta rhythm dissociates grid cell spatial periodicity from directional tuning. Science 332:595-599. CrossRef Medline

Brown MW, Banks PJ (2015) In search of a recognition memory engram. Neurosci Biobehav Rev 50:12-28. CrossRef Medline

Burke SN, Maurer AP, Hartzell AL, Nematollahi S, Uprety A, Wallace JL, Barnes CA (2012) Representation of three-dimensional objects by the rat perirhinal cortex. Hippocampus 22:2032-2044. CrossRef Medline

Burwell RD, Amaral DG (1998) Cortical afferents of the perirhinal, postrhinal, and entorhinal cortices of the rat. J Comp Neurol 398:179-205. CrossRef Medline

Burwell RD, Witter MP, Amaral DG (1995) Perirhinal and postrhinal cortices of the rat: a review of the neuroanatomical literature and comparison with findings from the monkey brain. Hippocampus 5:390-408. CrossRef Medline

Carpenter F, Manson D, Jeffery K, Burgess N, Barry C (2015) Grid cells form a global representation of connected environments. Curr Biol 25: 1176-1182. CrossRef Medline

Davachi L (2006) Item, context and relational episodic encoding in humans. Curr Opin Neurobiol 16:693-700. CrossRef Medline

Deshmukh SS, Knierim JJ (2011) Representation of non-spatial and spatial information in the lateral entorhinal cortex. Front Behav Neurosci 5:69. CrossRef Medline

Deshmukh SS, Johnson JL, Knierim JJ (2012) Perirhinal cortex represents nonspatial, but not spatial, information in rats foraging in the presence of objects: comparison with lateral entorhinal cortex. Hippocampus 22: 2045-2058. CrossRef Medline

Diana RA, Yonelinas AP, Ranganath C (2007) Imaging recollection and familiarity in the medial temporal lobe: a three-component model. Trends Cogn Sci 11:379-386. CrossRef Medline

Eichenbaum H, Yonelinas AP, Ranganath C (2007) The medial temporal lobe and recognition memory. Annu Rev Neurosci 30:123-152. CrossRef Medline

Furtak SC, Ahmed OJ, Burwell RD (2012) Single neuron activity and theta modulation in postrhinal cortex during visual object discrimination. Neuron 76:976-988. CrossRef Medline

Hafting T, Fyhn M, Molden S, Moser MB, Moser EI (2005) Microstructure of a spatial map in the entorhinal cortex. Nature 436:801-806. CrossRef Medline

Hargreaves EL, Rao G, Lee I, Knierim JJ (2005) Major dissociation between medial and lateral entorhinal input to dorsal hippocampus. Science 308: 1792-1794. CrossRef Medline

Igarashi KM, Lu L, Colgin LL, Moser MB, Moser EI (2014) Coordination of entorhinal-hippocampal ensemble activity during associative learning. Nature 510:143-147. CrossRef Medline

Kerr KM, Agster KL, Furtak SC, Burwell RD (2007) Functional neuroanatomy of the parahippocampal region: the lateral and medial entorhinal areas. Hippocampus 17:697-708. CrossRef Medline

Knierim JJ, Neunuebel JP, Deshmukh SS (2013) Functional correlates of the lateral and medial entorhinal cortex: objects, path integration and localglobal reference frames. Philos Trans R Soc Lond B Biol Sci 369:20130369. CrossRef Medline

Komorowski RW, Manns JR, Eichenbaum H (2009) Robust conjunctive item-place coding by hippocampal neurons parallels learning what happens. J Neurosci 29:9918-9929. CrossRef Medline

Kriegeskorte N, Mur M, Bandettini P (2008) Representational similarity analysis: connecting the branches of systems neuroscience. Front Syst Neurosci 2:4. CrossRef Medline

Lee I, Yoganarasimha D, Rao G, Knierim JJ (2004) Comparison of population coherence of place cells in hippocampal subfields CA1 and CA3. Nature 430:456-459. CrossRef Medline

Leutgeb S, Leutgeb JK, Treves A, Moser MB, Moser EI (2004) Distinct ensemble codes in hippocampal areas CA3 and CA1. Science 305: 1295-1298. CrossRef Medline

Marozzi E, Ginzberg LL, Alenda A, Jeffery KJ (2015) Purely translational 
realignment in grid cell firing patterns following nonmetric context change. Cereb Cortex 25:4619-4627. CrossRef Medline

McKenzie S, Frank AJ, Kinsky NR, Porter B, Rivière PD, Eichenbaum H (2014) Hippocampal representation of related and opposing memories develop within distinct, hierarchically organized neural schemas. Neuron 83:202-215. CrossRef Medline

Mizuseki K, Buzsáki G (2013) Preconfigured, skewed distribution of firing rates in the hippocampus and entorhinal cortex. Cell Rep 4:1010-1021. CrossRef Medline

Morrissey MD, Takehara-Nishiuchi K (2014) Diversity of mnemonic function within the entorhinal cortex: a meta-analysis of rodent behavioral studies. Neurobiol Learn Mem 115:95-107. CrossRef Medline

Moser EI, Moser MB (2013) Grid cells and neural coding in high-end cortices. Neuron 80:765-774. CrossRef Medline

Moser EI, Moser MB, Roudi Y (2013) Network mechanisms of grid cells. Philos Trans R Soc Lond B Biol Sci 369:20120511. CrossRef Medline

Neunuebel JP, Yoganarasimha D, Rao G, Knierim JJ (2013) Conflicts between local and global spatial frameworks dissociate neural representations of the lateral and medial entorhinal cortex. J Neurosci 33: 9246-9258. CrossRef Medline

Paxinos G, Watson C (2007) The rat brain in stereotaxic coordinates, Ed 6. San Diego: Academic Press.

Rigotti M, Barak O, Warden MR, Wang XJ, Daw ND, Miller EK, Fusi S (2013) The importance of mixed selectivity in complex cognitive tasks. Nature 497:585-590. CrossRef Medline

Sargolini F, Fyhn M, Hafting T, McNaughton BL, Witter MP, Moser MB, Moser EI (2006) Conjunctive representation of position, direction, and velocity in entorhinal cortex. Science 312:758-762. CrossRef Medline

Sasaki T, Leutgeb S, Leutgeb JK (2015) Spatial and memory circuits in the medial entorhinal cortex. Curr Opin Neurobiol 32:16-23. CrossRef Medline

Shamir M (2014) Emerging principles of population coding: in search for the neural code. Curr Opin Neurobiol 25:140-148. CrossRef Medline

Solstad T, Boccara CN, Kropff E, Moser MB, Moser EI (2008) Representation of geometric borders in the entorhinal cortex. Science 322: 1865-1868. CrossRef Medline

Suzuki WA, Amaral DG (1994) Perirhinal and parahippocampal cortices of the macaque monkey: cortical afferents. J Comp Neurol 350:497-533. CrossRef Medline

Suzuki WA, Miller EK, Desimone R (1997) Object and place memory in the macaque entorhinal cortex. J Neurophysiol 78:1062-1081. Medline

Tsao A, Moser MB, Moser EI (2013) Traces of experience in the lateral entorhinal cortex. Curr Biol 23:399-405. CrossRef Medline

van Strien NM, Cappaert NL, Witter MP (2009) The anatomy of memory: an interactive overview of the parahippocampal-hippocampal network. Nat Rev Neurosci 10:272-282. CrossRef Medline

Witter MP, Groenewegen HJ, Lopes da Silva FH, Lohman AH (1989) Functional organization of the extrinsic and intrinsic circuitry of the parahippocampal region. Prog Neurobiol 33:161-253. CrossRef Medline

Witter MP, Canto CB, Couey JJ, Koganezawa N, O’Reilly KC (2014) Architecture of spatial circuits in the hippocampus. Philos Trans R Soc Lond B Biol Sci 369:20120515. CrossRef Medline

Yanike M, Wirth S, Smith AC, Brown EN, Suzuki WA (2009) Comparison of associative learning-related signals in the macaque perirhinal cortex and hippocampus. Cereb Cortex 19:1064-1078. CrossRef Medline

Young BJ, Otto T, Fox GD, Eichenbaum H (1997) Memory representation within the parahippocampal region. J Neurosci 17:5183-5195. Medline 